\title{
Testing the downscaling ability of a one-way nested regional climate model in regions of complex topography
}

Received: 19 June 2003/ Accepted: 11 March 2004/Published online: 20 December 2005

(C) Springer-Verlag 2005

\begin{abstract}
The downscaling ability of a one-way nested regional climate model (RCM) is evaluated over a region subjected to strong surface forcing: the west of North America. The sensitivity of the results to the horizontal resolution jump and updating frequency of the lateral boundary conditions are also evaluated. In order to accomplish this, a perfect-model approach nicknamed the Big-Brother Experiment (BBE) was followed. The experimental protocol consists of first establishing a virtual-reality reference climate over a fairly large area by using the Canadian RCM with grid spacing of $45 \mathrm{~km}$ nested within NCEP analyses. The resolution of the simulated climate is then degraded to resemble that of operational general circulation models (GCM) or observation analyses by removing small scales; the filtered fields are then used to drive the same regional model, but over a smaller sub-area. This set-up permits a comparison between two simulations of the same RCM over a common region. The Big-Brother Experiment has been carried out for four winter months over the west coast of North America. The results show that complex topography and coastline have a strong positive impact on the downscaling ability of the one-way nesting technique. These surface forcings, found to be responsible for a large part of small-scale climate features, act primarily locally and yield good climate reproducibility. Precipitation over the Rocky Mountains region is a field in which such effect is found and for which the nesting
\end{abstract}

Figure legends were missing in original article. Climate Dynamics (2005) 23: 473-493. The complete article is given here. DOI: 10.1007/s00382-004-0438-5 http://dx.doi.org/10.1007/s00382-004$0438-5$

S. Antic $\cdot$ R. Laprise $\cdot$ R. de Elía $(\bowtie)$

Département des sciences de la Terre et de l'Atmosphére, Université du Québec á Montréal, 550 Sherbrooke St. West, 19th Floor, Montréal, Québec Canada H3A 1B9

E-mail: relia@sca.uqam.ca

B. Denis

Canadian Meteorological Centre,

Meteorological Service of Canada, Dorval, Québec Canada technique displays significant downscaling ability. The best downscaling ability is obtained when the ratio of spatial resolution between the nested model and the nesting fields is less than 12 , and when the update frequency is more than twice a day. Decreasing the spatial resolution jump from a ratio of 12 to six has more benefits on the climate reproducibility than a reduction of spatial resolution jump from two to one. Also, it is found that an update frequency of four times a day leads to a better downscaling than twice a day when a ratio of spatial resolution of one is used. On the other hand, no improvement was found by using high-temporal resolution when the driving fields were degraded in terms of spatial resolution.

\section{Introduction}

The resolution of present-day general circulation models (GCMs) is insufficient to resolve mesoscale atmospheric circulations, for example, those forced by small-scale topography or surface heterogeneities, and sub-synoptic phenomena. Because the computing time required for running a GCM increases approximately with the third or fourth power of the increase in the horizontal resolution, running a uniform-resolution global climate model over high-resolution grid mesh has an unacceptable computational cost for the lengths of time required for climate-change simulations. Since climate impact studies would benefit from such small-scale information, some methods of "downscaling" output from coarsegrid GCMs are now in use. One such technique is the one-way nesting and is classified as a dynamical downscaling technique (as opposed to statistical downscaling techniques). With the one-way nesting, the downscaling is achieved over a selected region by nesting a highresolution atmospheric regional-climate model (RCM) within a global GCM, or within global analyses of atmospheric observations e.g. Jones et al. 1995; Walsh 
and McGregor 1995; Laprise et al. (1997, 2000); Takle et al. 1999; Hong and Leetmaa 1999; Leung et al. 1999; de Elía et al. 2001.

Since RCMs are limited-area models they have to be forced by nesting information at the perimeter of the regional domain. The most commonly used nesting strategy is the one-way nesting technique designed by Davies (1976). This one-way approach does not permit feedback between a regional climate model and driving data. On the one hand this is an advantage in that the global model does not have to be integrated each time a RCM is applied. On the other hand, the absence of interactions at the boundaries may have negative impacts on the simulated climatology. Several issues regarding the one-way nested RCMs are summarised and explained in the work of Giorgi and Mearns (1999) and Denis et al. (2002b).

The primary aim of an RCM used as a climate downscaling technique is to generate a realistic regional climate having fine-scale features at scales that are absent in the initial and lateral boundary conditions (LBC) used to initialise and nest it. The affordable fine resolution over a limited area allows better representation of the surface forcings such as topography, coastlines, inland water or land-surface characteristics, which are the main source of fine-scale features (e.g. Jones et al. 1995; Leung and Ghan 1998; Laprise et al. 1997). Hydrodynamic instabilities and nonlinear processes such as stretching, folding and stirring by meanders in atmospheric circulation are also better represented on the high-resolution gridmesh and can develop fine-scale structures even in the absence of fine-scale surface forcings.

Similarly to global models, RCMs are susceptible to a variety of error sources. Numerical approximation, subgrid-scale physical parametrisation and limited resolutions in space and time are sources of errors for RCMs as well as for GCMs. In addition, a number of issues concerning the use of one-way nesting techniques constitute additional sources of errors or climate drift in RCMs. Consequently, the Working Group on Numerical Experimentation (WGNE) of the World Climate Research Programme (WCRP) expressed scepticism in one-way nested RCMs as a climate downscaling technique (CAS/JSC WGNE, 1999, 2000). They pointed out the need for developing an experiment that could estimate objectively the downscaling ability of one-way nested RCMs.

Recognising the suggestion of WGNE, Denis et al. (2002b) designed an idealised experimental protocol, nicknamed the Big-Brother Experiment (BBE), in order to validate the one-way nesting strategy of regional climate models. As a first step, a high-resolution RCM was used to establish a virtual-reality reference climate over a large domain (Big-Brother simulation, BB). Thereafter, the output of $\mathrm{BB}$ was degraded toward the resolution of contemporary global models or objective analyses by filtering small scales in order to imitate the effect of lowresolution nesting data. The resulting data were used for nesting the same RCM but over a smaller sub-area of the BB domain (Little-Brother simulation, LB). Comparing winter climate statistics over the eastern part of North America, Denis et al. (2002b, 2003) found that the LB one-way nested RCM regenerated rather well smallscale features of the BB despite the fact that such features were absent in the initial and lateral boundary conditions supplied for the LB.

The present work exploits the experimental methodology presented in Denis et al. (2002b, 2003) over the west coast of North America, a region with topographical forcing of great magnitude. As can be seen in Fig. 1, the computational domain of the Little Brother encompasses extensive continuous influence of open water (northeast of Pacific Ocean), sea ice (Hudson Bay), mountain ranges (the Rocky Mountains complex) and flat land (the Canadian northwestern Territories, Canadian and North-West of United States Prairies). The objective of this study is the investigation of the downscaling ability and sensitivity of the one-way nested Canadian regional-climate model (CRCM) to the horizontal resolution jump between LBC and RCM, as well as updating frequency of the lateral boundary conditions, in a region with strong surface forcing. This study will complement the investigation of Denis et al. (2002b, 2003) whose domain was situated over the coast of northeastern North America, a region with much weaker topographic forcing.

The work is organised in three main sections. In Sect. 2, a brief description of CRCM and experimental design for validating downscaling ability is given. Section 3 contains the simulated results and discussion, and in Sect. 4 the conclusions are presented.

\section{The CRCM and experimental design}

\subsection{Brief model description}

The Canadian regional climate model (CRCM) used in these experiments is a limited-area model based on fully elastic nonhydrostatic equations, solved with a semiLagrangian and semi-implicit time-marching scheme (see Bergeron et al. 1994; Laprise et al. 1997). Horizontal representation of the fields uses an Arakawa C-grid on a polar-stereographic projection; in the vertical, Gal-Chen scaled-height terrain-following coordinates are used. The time step and grid-point spacing used in the experiment were $15 \mathrm{~min}$ and $45 \mathrm{~km}$, respectively. The subgrid-scale physical parametrisation package is imported from the second-generation Canadian GCM (McFarlane et al. 1992) and adapted for use in the CRCM (Caya and Laprise 1999); the version used here includes the Kain and Fritsch (1990) deep cumulus convection scheme. The sea-surface temperature (SST) is imposed by linear time interpolation of the monthly mean climatological data. The nesting method used to drive the CRCM was inspired by Davies (1976) and refined by Robert and Yakimiw (1986) and Yakimiw and Robert (1990). The nesting is done in the nine-point 
Fig. 1 The computational domains for Big Brother (BB) and Little Brother (LB). Land-sea-ice mask and topographic height are shown only for the smaller domain. Land,sea and marine ice areas are presented in dark, medium and light gray, respectively. Three separate regions (ocean, mountains and flat land) are used for different statistics calculations. Contours of the topography are $[100,200,300$, $400, \ldots .1,000,1,200,1,400$, $1,600,2,000 \ldots ..] \mathrm{m}$

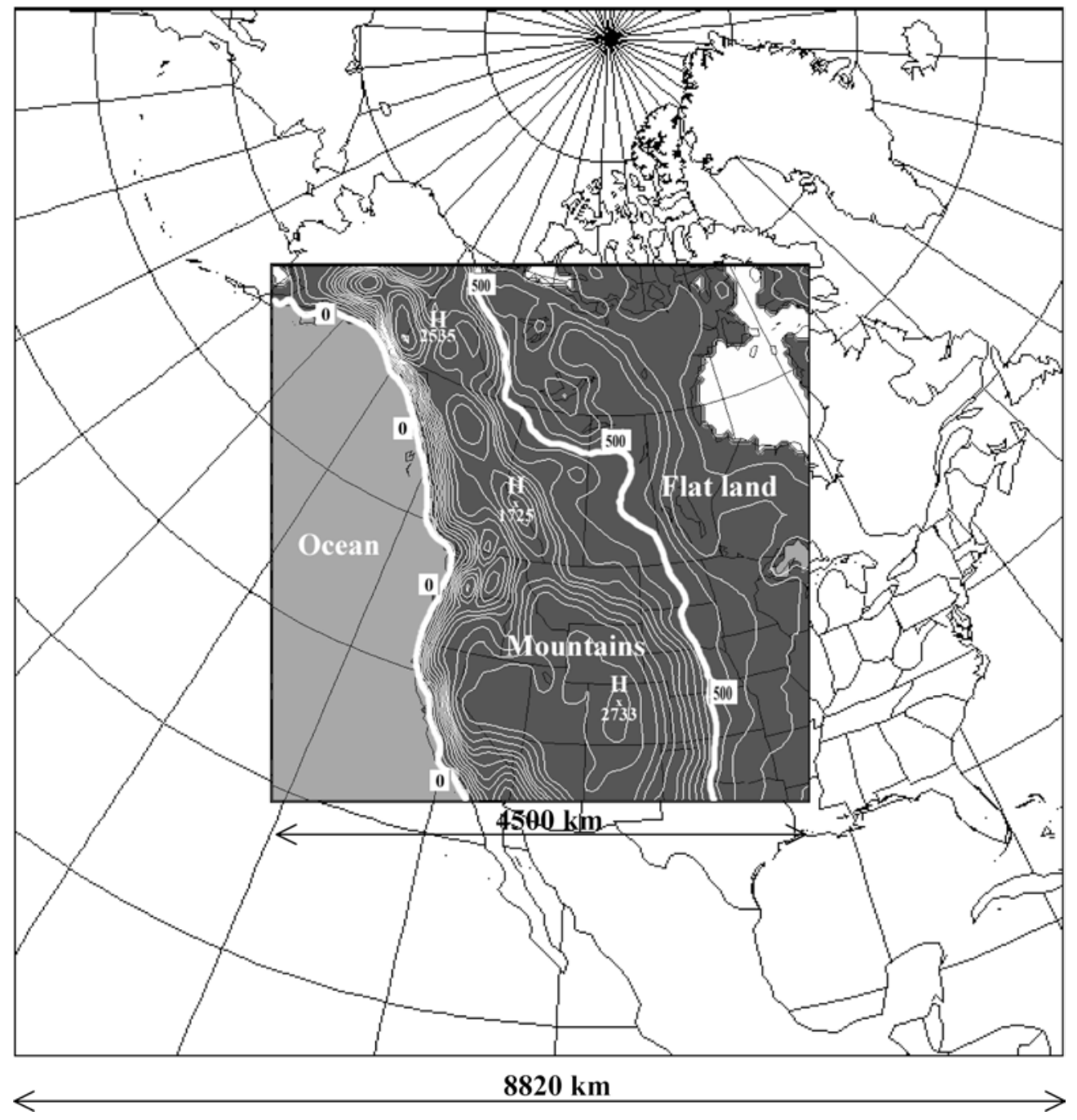

wide region around the perimeter of the computational domain (buffer zone), in which the horizontal velocities are relaxed toward those in the driving model and zero is imposed to the vertical velocity; all time-dependent variables are imposed at the perimeter of the CRCM from the nesting data.

2.2 The big-brother experiment and details of the experimental set-up

To achieve the objectives of this work, the experiment named Big-Brother was used following the methodology described in the Denis et al. (2002b) and Denis et al. (2003). It is briefly summarised below.

A large-domain high-resolution version of Canadian RCM, nested by global objective analyses NCEP (see Hong and Leetmaa 1999; Takle et al. 1999 for a reference) archived every $12 \mathrm{~h}$, is used to establish a virtual climate over a large domain (Big-Brother), whose size corresponds to approximately $9000 \mathrm{~km}^{2}$. This simulation was degraded by filtering short scales and used for nesting the same CRCM but over a smaller domain of approximately $4500 \mathrm{~km}^{2}$, which is embedded in the BigBrother domain as shown in Fig. 1 (Little Brother). Comparison between the climate statistics of the BB and LB over the smaller domain gives an assessment of downscaling ability of the CRCM. Furthermore, differences in two climates can thus be attributed unambiguously to errors associated with the one-way nesting strategy, and not to model errors or physical parametrisations nor to quality of driving data. Figure 2 shows schematically the Big-Brother experimental protocol.

The computational domain for this study is centered over western North America. Topographical heights and land-sea-ice mask for simulations are presented in Fig. 1. The size of the BB domain was chosen to ensure that lateral buffer zone was as far as possible from the zone of interest (domain of the LB). In this regard, following recommendations of Warner et al. (1997) the lateral boundaries are separated in each direction from the LB domain by a distance equal to one-half of size of the LB domain. This seems to guarantee that the average properties of the flow become independent of the distance to the boundaries in the area of interest. This is important in this experiment because the Little Brother 


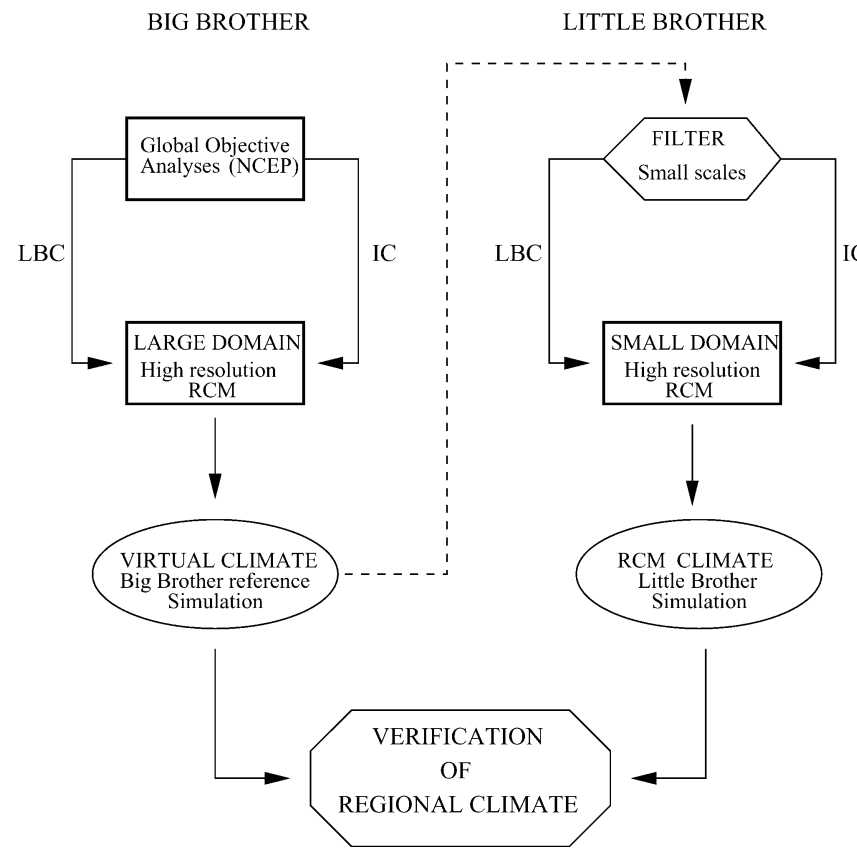

Fig. 2 Flow chart of the BB experimental protocol. The initial conditions (IC) and lateral boundary conditions $(L B C)$ for nesting the large-domain BB are obtained from NCEP analyses, while those for nesting small-domain LB are obtained by filtering small scales in the Big-Brother reference simulation. Climate validation is performed over the Little-Brother domain, excluding the lateral nesting zone

is supposed to reproduce a well-developed flow, as would do if nested in a high-resolution global model. Under this selection of domains, even changes in the nesting frequency of the Big Brother do not seem to affect the reproduction abilities of the Little Brother.

The simulations were executed for four February months $(1990,1991,1993,1994)$ with five days of spinup. The initial surface and atmospheric conditions were set at the beginning of the simulations, and the atmospheric LBC were updated every 3, 6 or $12 \mathrm{~h}$, depending on the experiment; these updating intervals correspond to update frequencies of 8,4 or 2 times per day (U8, U4 and $\mathrm{U} 2$ ), respectively. Linear time interpolation between these updating times was used to provide the nesting data required by the model at every time step.

In order to remove the small-scales features from the Big-Brother fields, the output was filtered using a 2D discrete cosine transformation as proposed by Denis et al. (2002a). In this study, filters J1, J6 and J12 have been employed and their response functions are presented in Fig. 3. The J1 "filter" keeps all wavelengths existing in $\mathrm{BB}$, while the $\mathrm{J} 6$ and $\mathrm{J} 12$ filters remove all wavelengths smaller than $500 \mathrm{~km}$ and $1000 \mathrm{~km}$, respectively. Filters J1, J6 and J12 correspond approximately to the T360, T60 and T30 spatial triangular spherical harmonic truncations of global spectral models and to a ratio of resolution (jumps) of 1,6 and 12 between nesting data and LB grid mesh. The simulations performed and described here are shown in Table 1. In

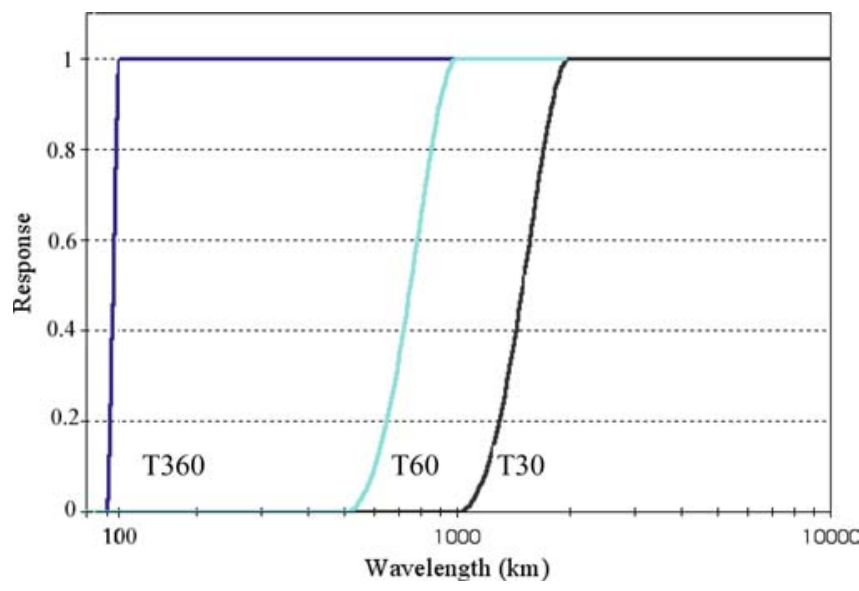

Fig. 3 Responses of the filters used to degrade the output of the $\mathrm{BB}$ simulation to provide low-resolution nesting data for LB simulations. Filters J6 and J12 are filtering completely wavelengths smaller than 500 and $1,000 \mathrm{~km}$, and fully preserve wavelengths greater than 1,000 and $2,000 \mathrm{~km}$, respectively. These filters correspond approximately to T60 and T30 spectral triangular spherical truncations. Filter J1 keep all wavelengths present in the Big-Brother, simulation which corresponds approximately to T360 spectral triangular spherical truncation

addition to the filtering discussed, the BB data were destaggered in the horizontal and interpolated from GalChen coordinates to pressure coordinates; the data were then filtered as $\mathrm{J} 1, \mathrm{~J} 6$ or $\mathrm{J} 12$; then the data were reinterpolated from pressure to Gal-Chen coordinates and re-staggered on the $\mathrm{C}$-grid. These interpolations act as a weak filtering, so in effect, even the $\mathrm{J} 1$ data are somewhat filtered compared to the BB.

\section{Results and discussion}

In order to achieve our objectives, the simulations are analysed from two points of view. First, attention is focused on the ability of the Little-Brother model to reproduce the high-resolution climate statistics of the Big-Brother (virtual reality) simulation when driven by data of resolution 6 times lower than the RCM (T360) at $3 \mathrm{~h}$ intervals. This first experiment corresponds to the

Table 1 List of experiments performed and discussed in this work

\begin{tabular}{llll}
\hline & \multicolumn{2}{l}{ Nesting interval } \\
\cline { 2 - 4 } Horizontal resolution & U8 (3 h) & U4 $(6$ h) & U2 (12 h) \\
\hline J1 (T360) & $\sqrt{ }$ & $\sqrt{ }$ & $\sqrt{ }$ \\
J6 (T60) & $\sqrt{ }$ & $\sqrt{ }$ & $\sqrt{ }$ \\
J12 (T30) & $\sqrt{ }$ & $\sqrt{ }$ & U
\end{tabular}

The J1, J6 and J12 resolutions have been employed for nesting small-domain LB; these correspond to T360, T60 and T30 triangular spherical truncations of global models, respectively. Lateral boundary conditions were updated every 3 , 6 , or 12 hours, corresponding to update frequencies of 8,4 or 2 update per day (U8, U4 or U2). The symbol $\sqrt{ }$ indicates experiments performed in this study 
J6/U8 experiment of Table 1. Second, the sensitivity of the Little-Brother simulation to update frequency and resolution jump is investigated. Finally, to evaluate the impact of complex surface conditions and the effect of different domain location, climate statistics will be compared with those presented in Denis et al. (2002b, 2003), whose domain was located over the eastern North America where topographical forcing is much weaker.

Each Little Brother simulation was run for 33 days and the first five days were discarded in the calculation of the climate statistics to reduce spin-up effects. In the forthcoming section simulated fields will be displayed over the entire Little-Brother computational domain, but the statistics will be computed excluding the 9-pointwide nesting zone.

\subsection{Climate downscaling ability}

The set of simulations have been analysed from a climatic point of view as follows: given a simulated horizontal field $\phi$, its monthly (short-term) climate mean value $\bar{\phi}$ has been computed by time-averaging over four February months. A measure of time variability is obtained in the usual way as transient-eddy standard deviation $\sigma=\sqrt{\overline{(\phi-\bar{\phi})}^{2}}$. In order to refine the analysis, the small-scale component $\phi_{s s}$. of a given $\phi$ field is extracted and considered separately from the total component field. In this paper, the fine-scale components considered for diagnostic purposes are those that survived a filtering J6 (Fig. 3).

In order to objectively evaluate the downscaling ability, spatial correlation coefficients $(\mathbf{R})$ between LittleBrother $\phi_{L}$ and Big-Brother fields $\phi_{B}$ are defined as

$R=\frac{\left\langle\varphi_{L}^{\prime} \varphi_{B}^{\prime}\right\rangle}{\sigma_{L} \sigma_{B}}$

where

$$
\begin{aligned}
\varphi^{\prime} & =\varphi-<\varphi>, \\
\sigma & =<\varphi^{\prime 2}>^{1 / 2},
\end{aligned}
$$

and $<>$ represents the horizontal average. This corresponds to Eq. 7 presented in Denis et al. (2002b).

In addition, a measure of the ability of the Little Brother to match the variability of the Big Brother is estimated by the ratio

$\Gamma=\frac{\sigma_{L}^{2}}{\sigma_{B}^{2}}$

where ${\sigma_{\mathrm{L}}}^{2}$ and ${\sigma_{\mathrm{B}}}^{2}$ are the variances of the Little and Big brother respectively. These statistics are also decomposed into total and small scales (more details can be found in Denis et al. (2002b, 2003)).

Examination of the ability of a one-way nested regional climate model to generate small-scale features that are absent in the initial and lateral boundary conditions is an important part of this study. For this pur- pose, the RCM nested in low-resolution data provided from Big-Brother filtered with J6 (T60) filter and the lateral boundary conditions updated every $3 \mathrm{~h}$ (experiment J6-U8) are considered in this section. Maps of the monthly mean and transient-eddy standard deviation of total and small-scale components of the field, for the Big and Little Brothers, will be presented in this section. The correlation coefficient and variance ratio will also be computed. In order to check how strongly the regeneration of fine scales depends on the surface characteristics, the correlation coefficients are calculated over three regions: the "ocean", the "flat land" and the "mountains" (see Fig. 1 for regions location).

\subsubsection{The sea level pressure}

Stationary Big-Brother and Little-Brother mean sea level pressure (slp) fields are presented in Fig. 4. The location of patterns is quite similar in both cases. The four-monthly mean of slp (see Fig. 4a) shows the climatological cyclonic activity in the Gulf of Alaska and strong on-shore flow from British Columbia to the state of Oregon. The small-scale stationary component of slp (see Fig. 4b) reflects the impact of the local mountain-induced ridging and lee troughing over the Alaska Range and the Rocky Mountains, but also the effect of downward extrapolation below ground level over the Columbia Mountains and the Front Range. The spatial correlation coefficient $R$ between $\mathrm{BB}$ and LB stationary total fields is $99 \%$ and ratio of spatial variance is $98 \%$. Stationary small-scale component of slp also exhibits high correlation $R=98 \%$ and $\Gamma=$ $96 \%$, which illustrates the ability of the Little Brother in regenerating the spatial variability of the Big Brother. Over the Pacific Ocean and the Rocky Mountains regions, LB is successfully regenerating the stationary slp $(R>98 \%)$; over the flat land, however, the correlation for small-scale features is lower $(R=$ $94 \%$ ) but still significant.

Transient-eddy standard deviations of slp are displayed in Fig. 5. These fields show strong variability of slp on the eastern side of the Rocky Mountains with maximum in western region of the North-West Territories, extending southward in Alberta through eastern Colorado. This maximum is a direct result of the regeneration of meteorological systems on lee side of mountains and penetration of cold air from the north. The Little Brother is simulating this activity with spatial correlation with Big Brother of $99 \%$ and $98 \%$, for total and small-scale features, respectively. Furthermore, the spatial variance of these transient-eddy fields are very well reproduced by the Little Brother $(\Gamma=100 \%$ and $\Gamma$ $=98 \%$ for the total and the small-scale components, respectively).

In order to obtain the assessment of climate downscaling ability in regions with complex surface conditions, it is useful to discuss the results shown in comparison with those presented in Denis et al. (2002b, 2003). From a climatological point of view, the distri- 
bution of mean slp represents the mean low-level circulation and the vertically integrated atmospheric mass distribution. Monthly mean and standard deviation of slp fields (Figs. 4 and 5) in comparison with the similarly simulated fields displayed in Figs. 9 and 10 and Figs. 811 of Denis et al. (2002b, 2003), show that the smalldomain LB is very successful in regenerating the refer-
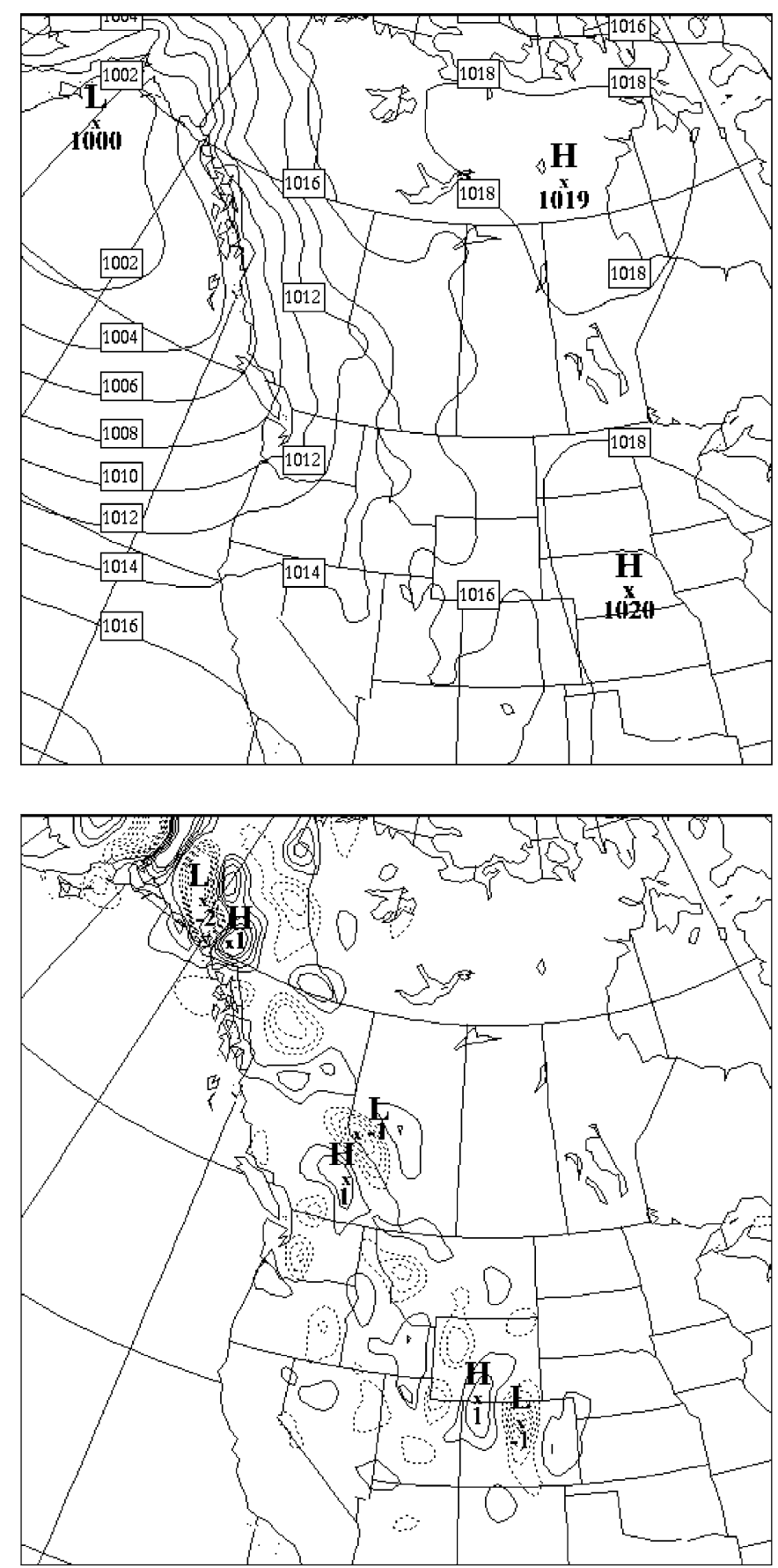

Fig. 4 a (upper panels) Four-monthly mean of sea-level pressure (slp). Isobars are contoured every $2 \mathrm{hPa}$. Correlation coefficients between $\mathrm{BB}$ on the left side and $\mathrm{LB}$ on the right side are $R=0.99$ for the entire domain; for ocean: $R=0.99$, land: $R=0.99$, flat land $R=0.99$, and mountains: $R=0.99$. b (lower panels) Stationary ence BB run. The correlation of $99 \%$ for monthly mean and transient slp in these J6-U8 experiments as in Denis et al. (2003) is not unexpected, since the sea level pressure is dominated by large-scale rather than small-scale features. Important improvement of correlation of the small-scale stationary and transient fields is found compared to Denis et al. (2002b): this is principally
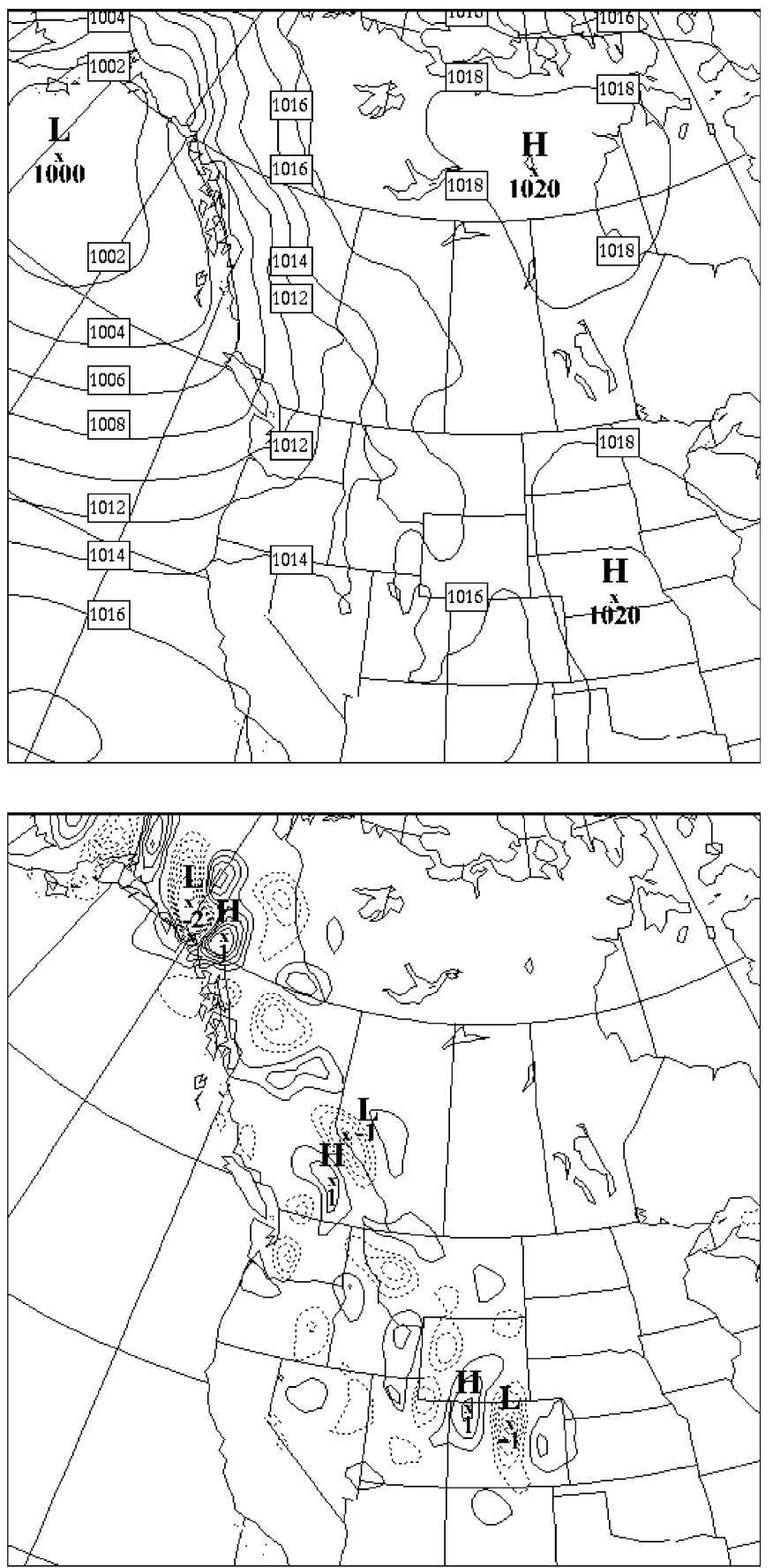

small-scale components of slp. Contours are $\{ \pm 1.0, \pm 0.8, \pm 0.6$, $\pm 0.4, \pm 0.2\} \mathrm{hPa}$. Correlation coefficients between BB on the left side and LB on the right side are $R=0.98$ for the entire domain; for ocean: $R=0.98$, land: $R=0.98$, flat land: $R=0.94$ and mountains: $R=0.99$ 

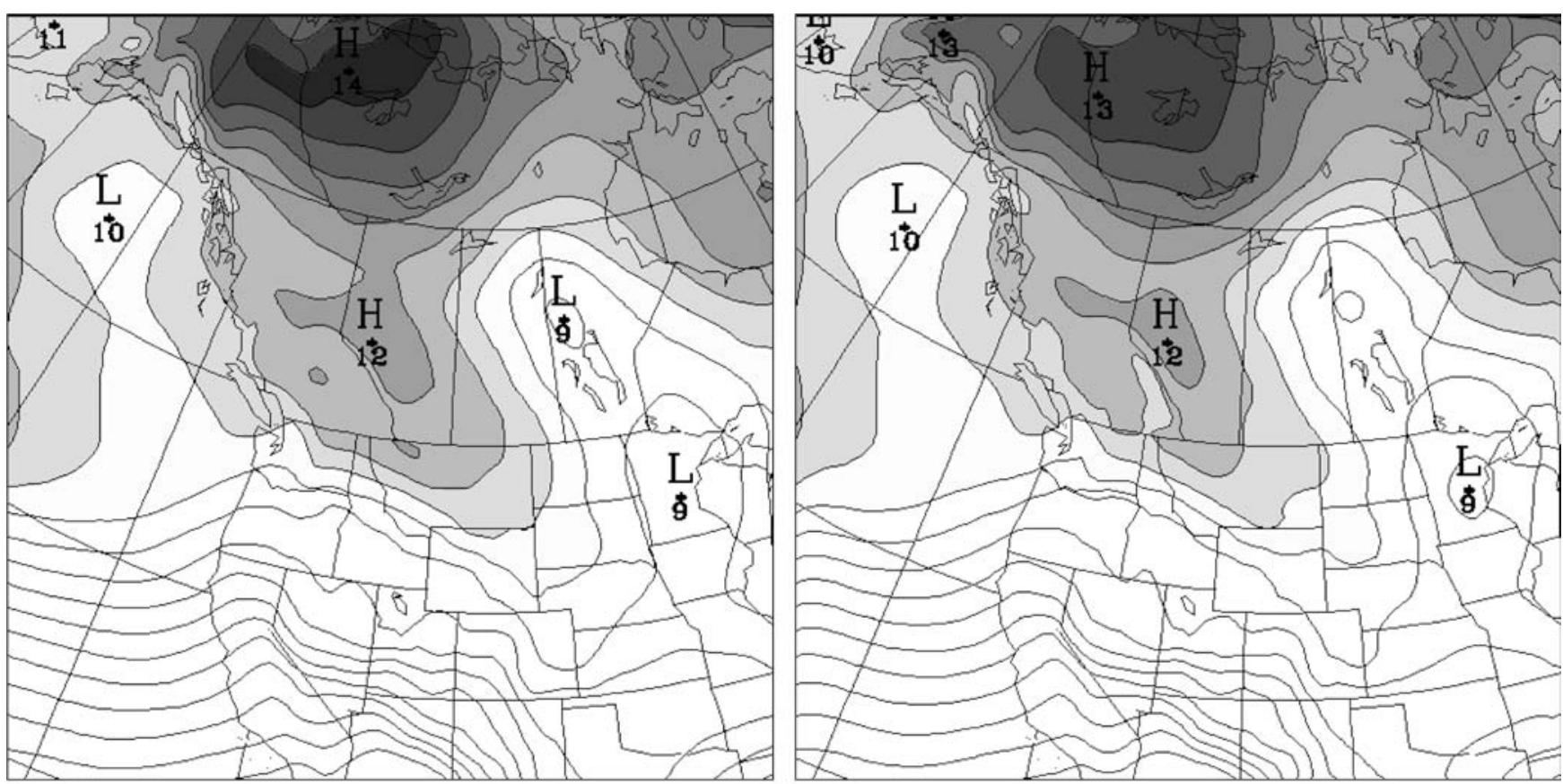

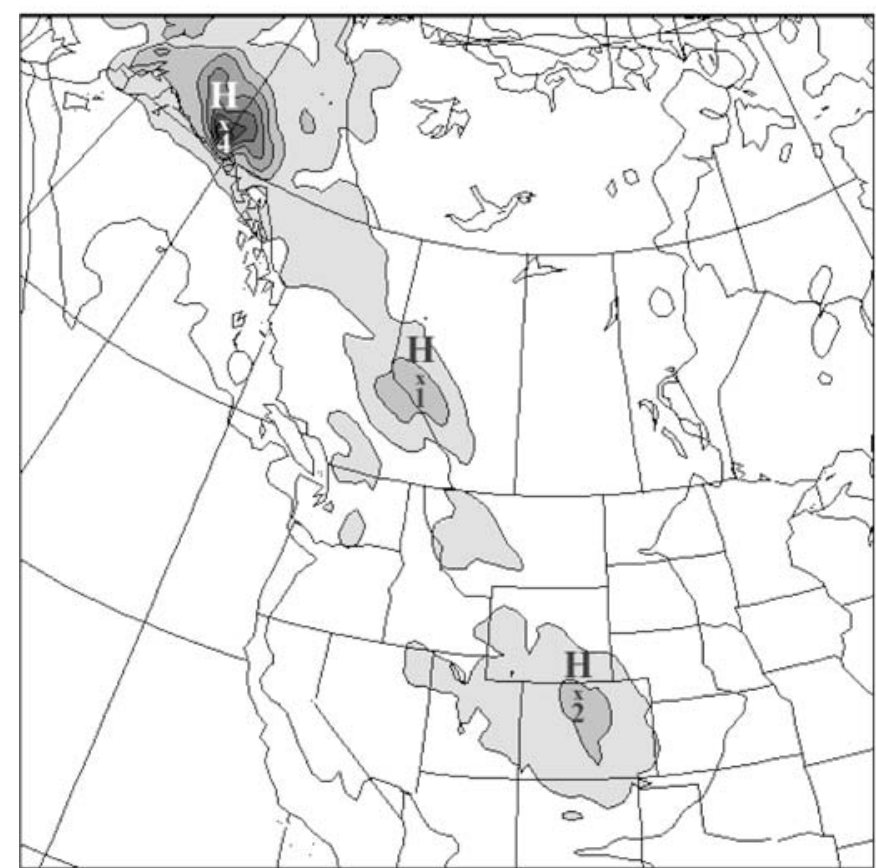

Fig. 5 a (upper panels) Transient-eddy standard deviation of slp. Contour interval is $0.5 \mathrm{hPa}$. Regions with values larger than $10 \mathrm{hPa}$ are shaded. Correlation coefficients between BB on the left side and LB on the right side are $R=0.99$ for the entire domain; for ocean: $R=0.99$, land: $R=0.99$, flat land: $R=0.99$ and mountains:

caused, however, by their use of single-month simulation in calculation of climate statistics.

\subsubsection{Precipitation field}

The precipitation field is the end product of complex interactions between the dynamics and physical

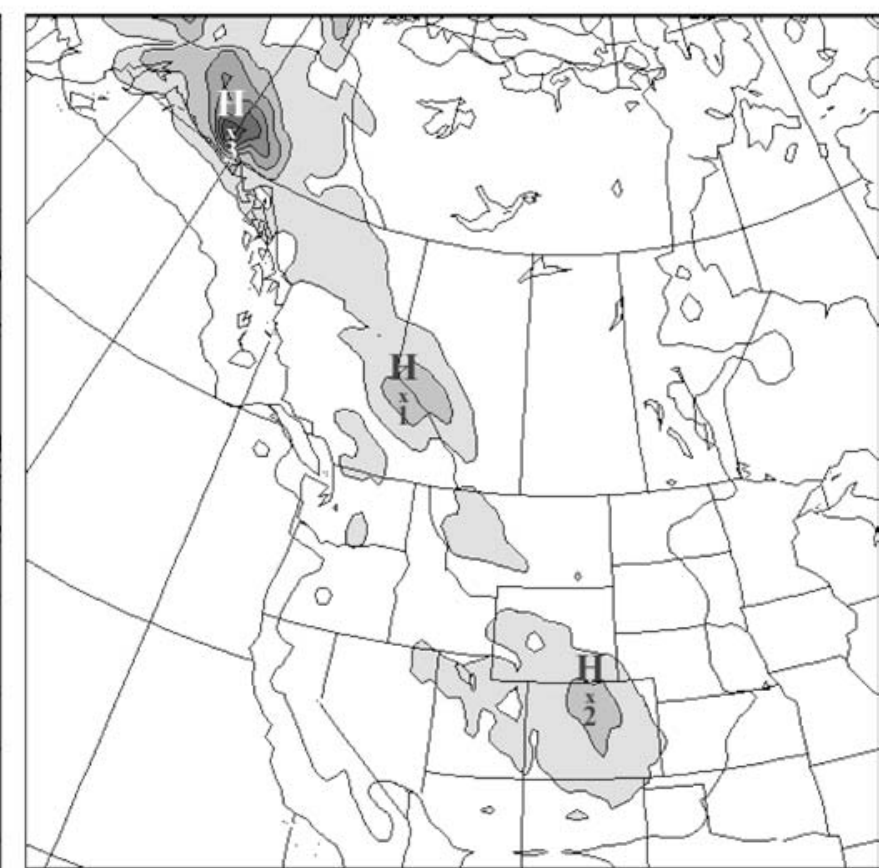

$R=0.99$. b (lower panels) Transient-eddy standard deviation of the small-scale component of slp. Contours are every $0.4 \mathrm{hPa}$. The regions larger than $0.8 \mathrm{hPa}$ are shaded. Correlation coefficients are $R=0.98$ for the entire domain; for ocean: $R=0.97$, land: $R=0.99$, flat land: $R=0.95$ and mountains: $R=0.99$

parametrisations used in the regional model. The capability of small-domain LB to reproduce this field will give an assessment of the one-way nested RCM downscaling ability. Figure 6a shows the narrow zone of strong precipitation on the windward side of the Rocky Mountains, which is very well reproduced by the LB $(R=99 \%$ for Rocky Mountains). The reduced LB precipitation in the relaxation zone is noticeable be- 
cause the vertical velocity, which has an important influence on precipitation, is set to be zero at the lateral boundaries in the CRCM. The stationary small-scale component (Fig. 6b) illustrates strong modulation of precipitation with topography. Ratio of spatial variability $(\Gamma=100 \%)$ and correlation coefficient $(R=$ $94 \%$ ) indicate the ability of LB to correctly generate small-scale precipitation.
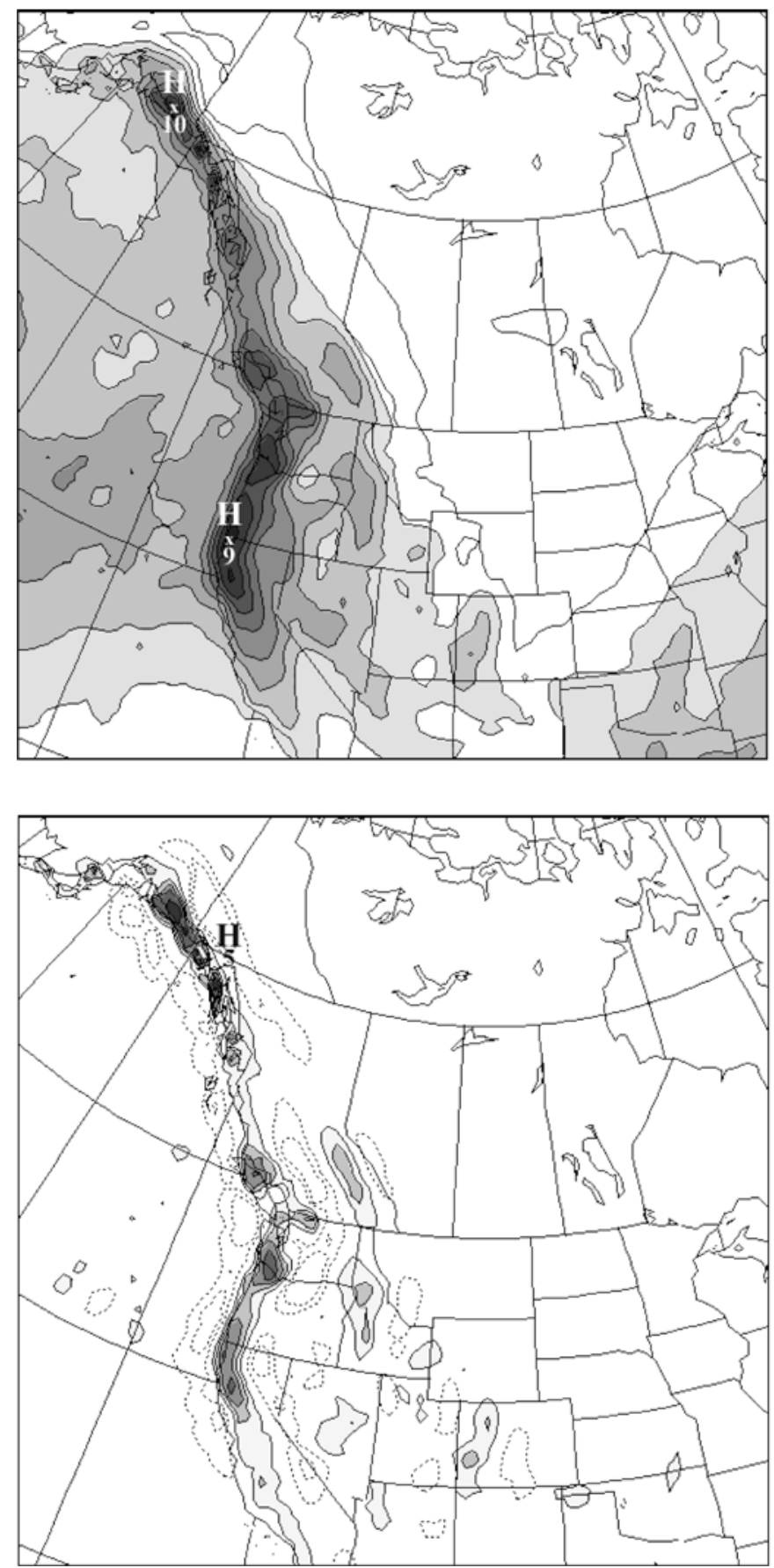

Fig. 6 a (upper panels) Monthly mean precipitation rate. Contours are every $1 \mathrm{~mm}$ /day. Regions larger than $2 \mathrm{~mm}$ /day are shaded. Correlation coefficients between BB on the left and LB on the right side are $R=0.98$ for the entire domain; for ocean: $R=0.94$, land: $R=0.99$, flat land: $R=0.99$ and mountains: $R=0.99$. b (lower
The transient parts of precipitation rate provide information regarding the passage of meteorological systems. Figure 7 indicates high spatial correlation $R=$ $96 \%$ between LB and BB transient-eddy standard deviation, both for the total field (Fig. 7a) and the smallscale component (Fig. 7b). An important part of transient precipitation is produced by small-scale phenomena, and patterns of maximum precipitation have similar
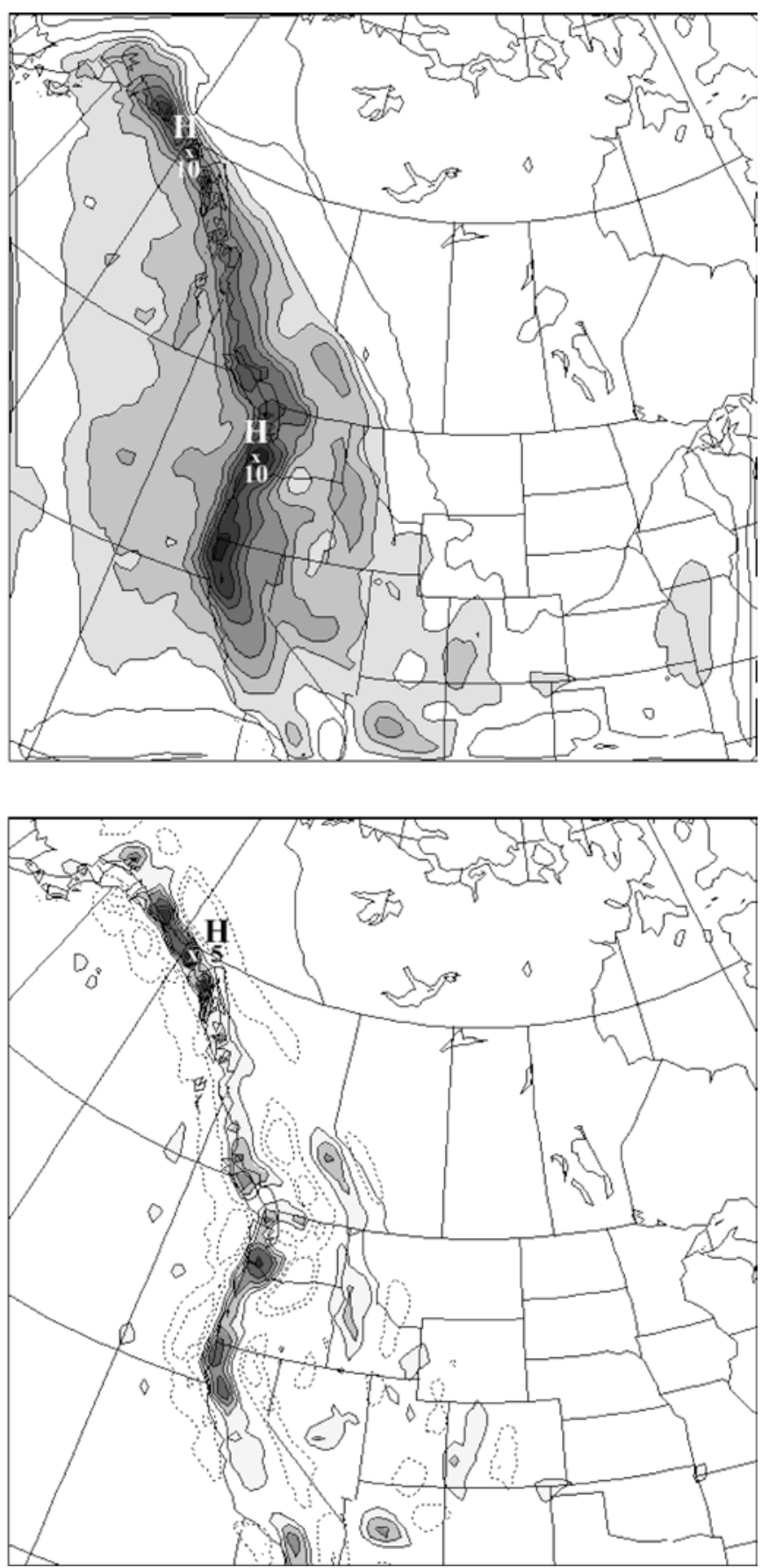

panels) Stationary small-scale component of the precipitation rate. Contours are $\{ \pm 5, \pm 3, \pm 1, \pm 0.5\} \mathrm{mm} /$ day. Regions shaded have values larger than $0.5 \mathrm{~mm}$ /day. Correlation coefficients are $R=0.94$ for the entire domain; for ocean: $R=0.90$, land: $R=0.96$, flat land: $R=0.95$ and mountains: $R=0.96$ 

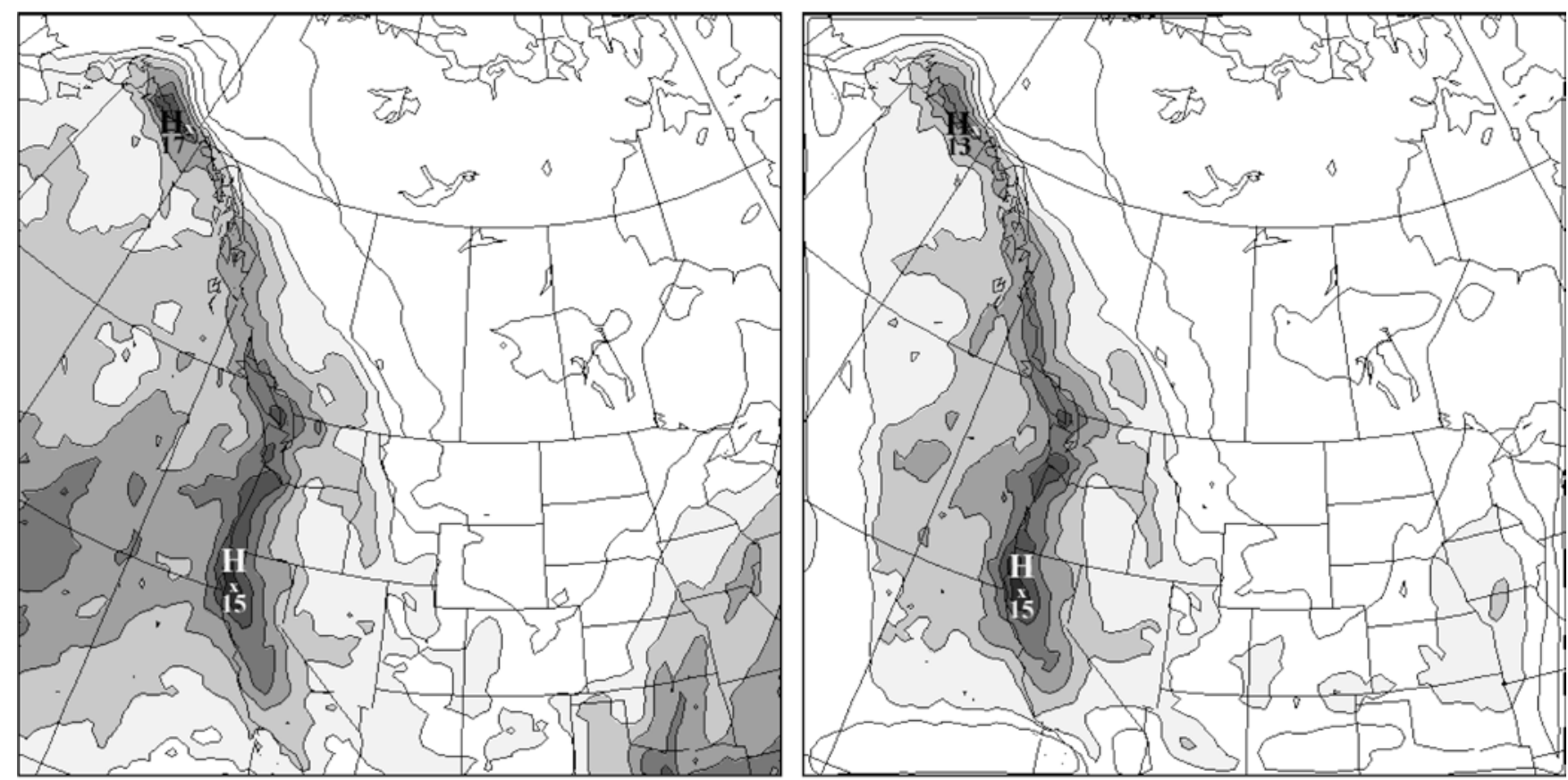

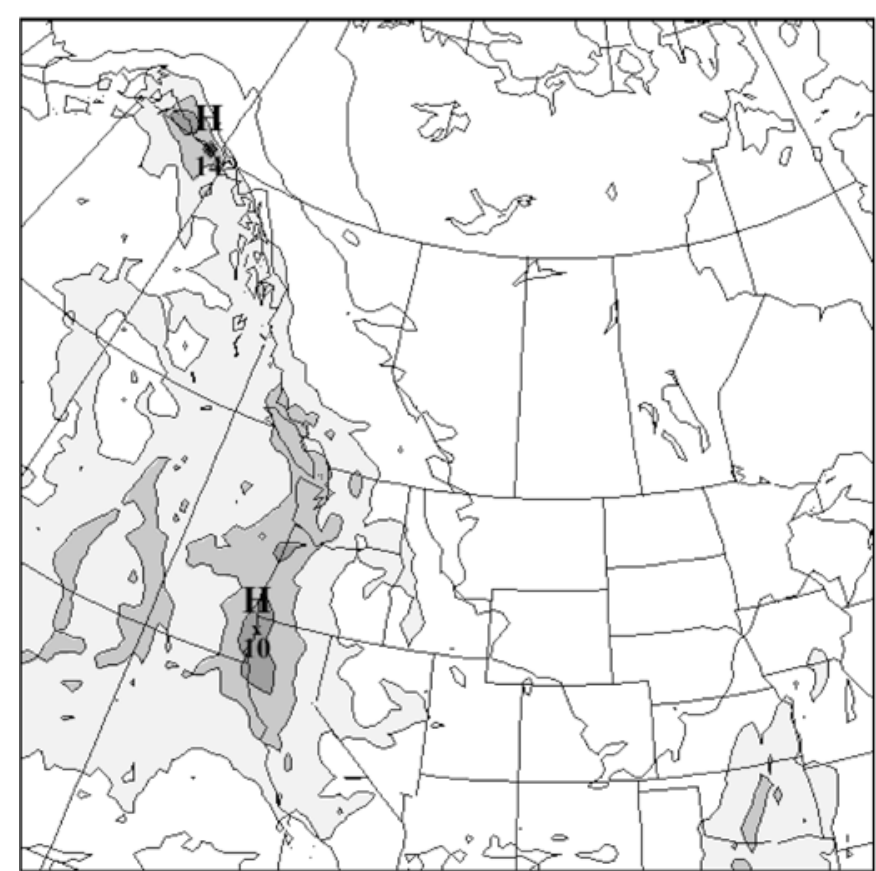

Fig. 7 a (upper panels) Transient-eddy standard deviation of precipitation rate. Contours are every $2 \mathrm{~mm} /$ day. Regions with values larger than $4 \mathrm{~mm} /$ day are shaded. Correlation coefficients between $\mathrm{BB}$ on the left side and $\mathrm{LB}$ on the right side are $R=0.96$ for the entire domain; for ocean: $R=0.86$, land: $R=0.97$, flat land: $R=0.96$ and mountains: $R=0.98$. b (lower panels) Transient-eddy

positions in Figs. 7a, 7b. The spatial variance ratio of LB to BB transient-eddy standard deviation field is $87 \%$ for total precipitation and $85 \%$ for the small-scale component. It is important to mention that, in regions of weak surface forcings, such as the ocean and flat land, the spatial correlation of small-scale transient eddies is a

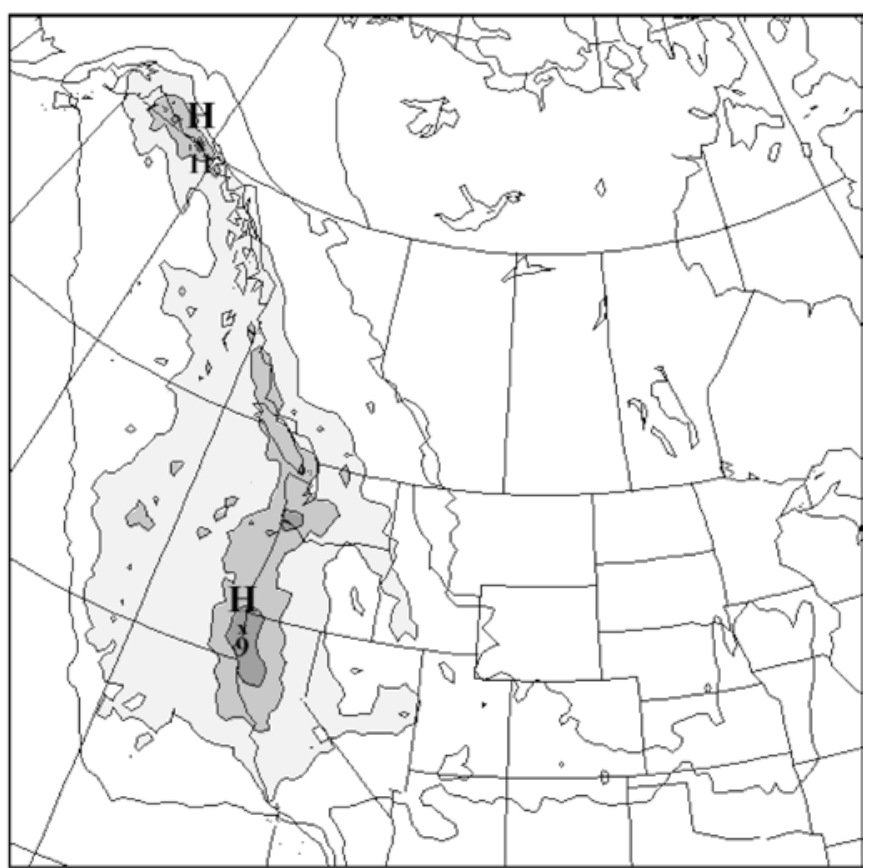

standard deviation of the small-scale component of precipitation rate. Contours are every $2 \mathrm{~mm} /$ day and region larger than $4 \mathrm{~mm} /$ day are shaded. Correlation coefficients are $R=0.96$ for the entire domain; for ocean: $R=0.83$, land: $R=0.96$, flat land: $R=0.95$ and mountains: $R=0.97$

little smaller than in the mountains region. In fact, the correlation is $83 \%$ and $95 \%$ for ocean and flat land, compared to $97 \%$ over the Rocky Mountains.

It is interesting to note that the precipitation is strongly modulated by the Rocky Mountains and coastline, and a large part of this field is in the small 
scales. The ability of Little Brother to regenerate the spatial distribution of the mean precipitation field (Fig. 6a) is in line with the same J6 (T60)-U8 experiment performed by Denis et al. (2003). It is perceptible that the complex surface conditions act as forcings of the stationary small-scale precipitation; this field shows significant improvement in correlation from $55 \%$ (on the east coast) in Fig. 13 of Denis et al. (2002b) compared to $94 \%$ in Fig. $6 \mathrm{~b}$. As for stationary fields, the topographical forcing has improved significantly
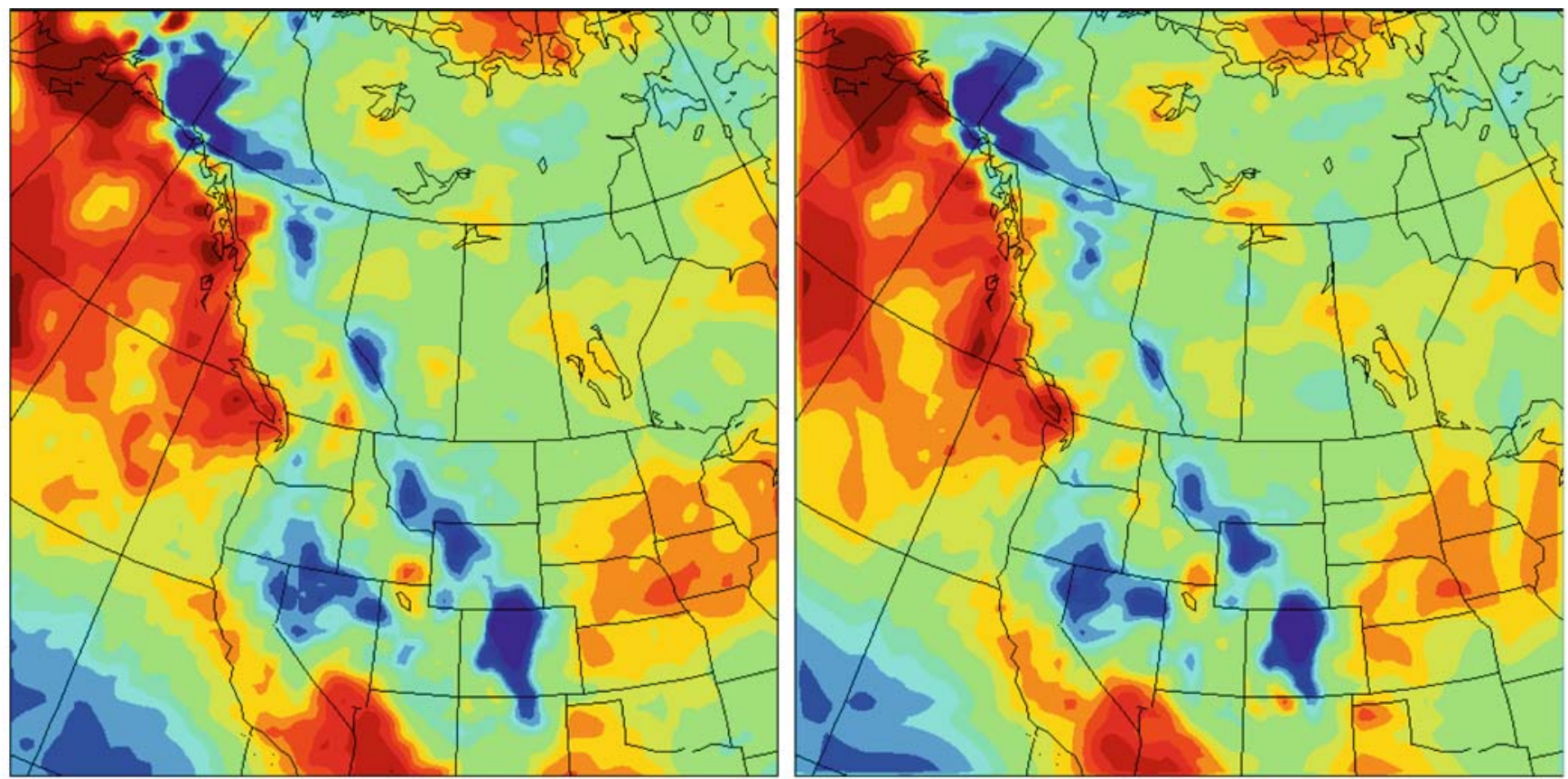

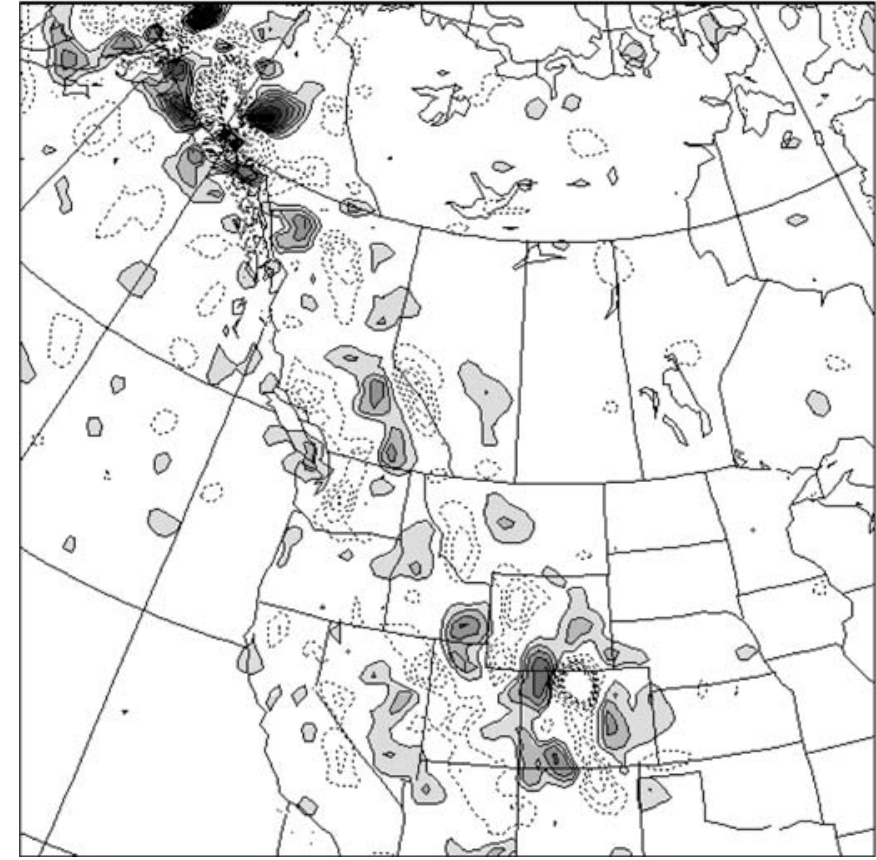

Fig. 8 a (upper panels) Monthly mean relative vorticity at $700 \mathrm{hPa}$. Contours are $\{ \pm 1.4, \pm 1.2, \pm 1.0, \pm 0.8, \pm 0.6, \pm 0.4$, $\pm 0.2\} \times 10^{-5} \mathrm{~s}^{-1}$. Red or blue regions correspond to positive or negative values, respectively. Correlation coefficients between BB and $\mathrm{LB}$ on the left and right side are $R=0.95$ for the entire domain; for ocean: $R=0.95$, land: $R=0.95$, flat land: $R=0.87$, and mountains: $\quad R=0.96$. b (lower panels) Stationary small-scale

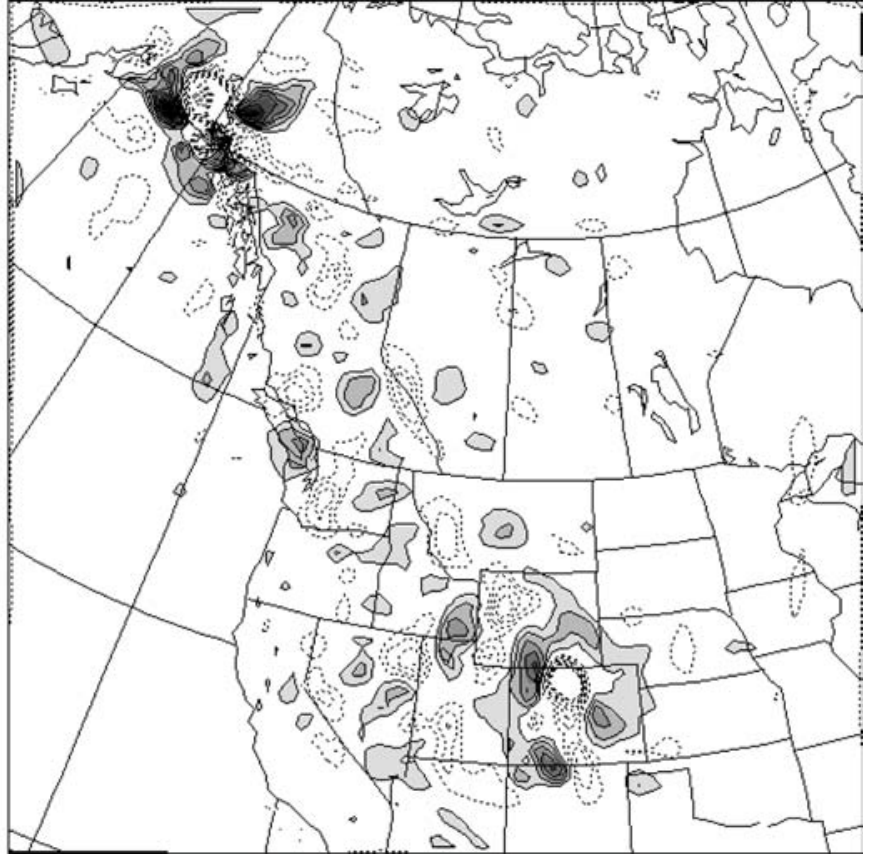

component of relative vorticity at $700 \mathrm{hPa}$. Contour intervals are $\{ \pm 1.4, \pm 1.2, \pm 1.0, \pm 0.8, \pm 0.6, \pm 0.4, \pm 0.2\} \times 10^{-5} \mathrm{~s}^{-1}$. Regions with values larger than $0.2 \times 10^{-5} \mathrm{~s}^{-1}$ are shaded. Contours with negative values are dotted. Correlation coefficients are $R=0.87$ for the entire domain; for ocean: $R=0.78$, land: $R=0.89$, flat land: $R=0.63$, and mountains: $R=0.91$ 
the downscaling of the transient precipitation comparing to Denis et al. (2003). Mean and transient precipitation fields simulated by a one-month simulation, presented in Figs. 13 and 14 of Denis et al. (2002b), explicitly demonstrate the importance of the number of months used in climate statistics calculations, especially over regions of weak surface forcing.
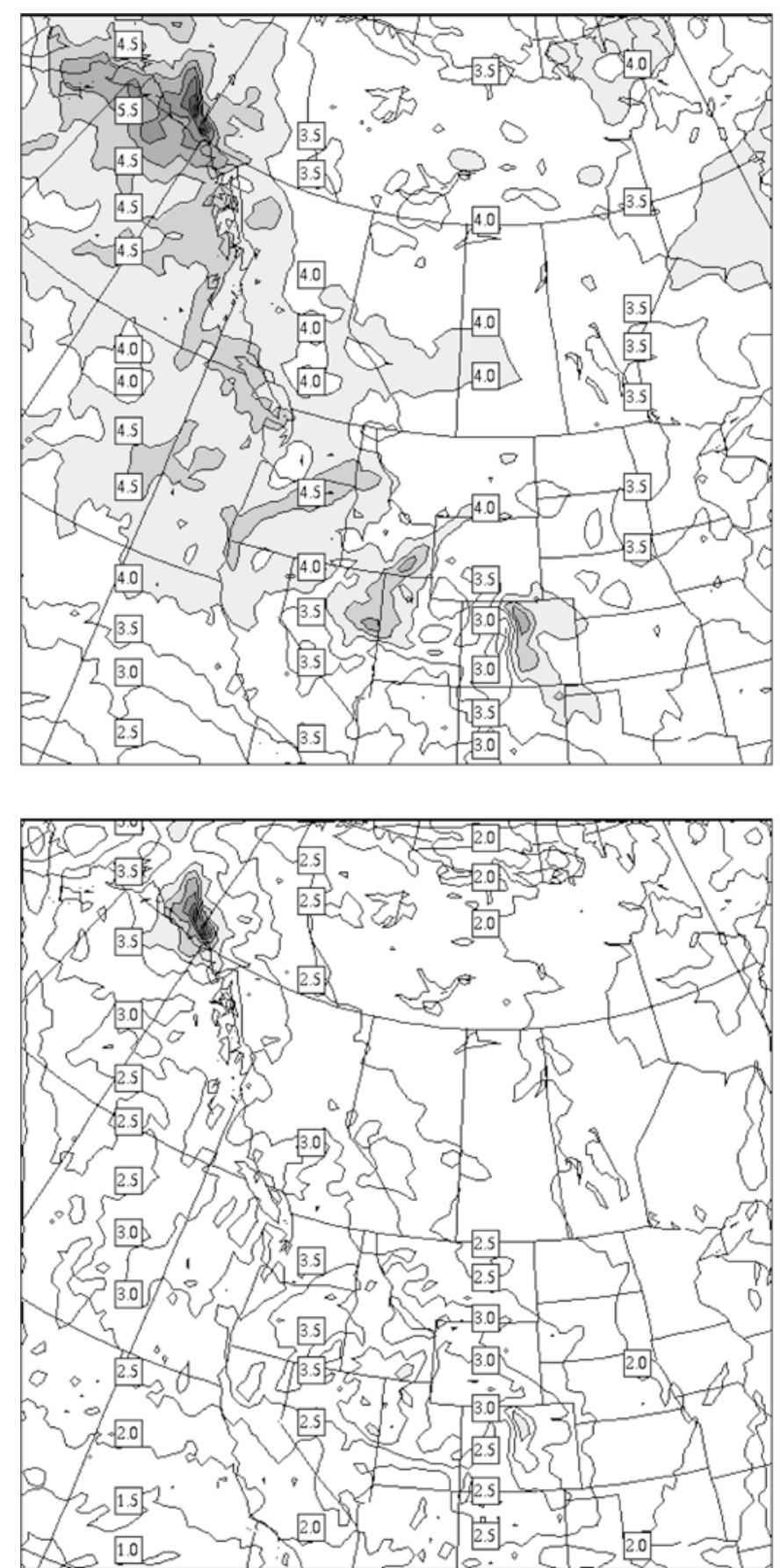

Fig. 9 a (upper panels) Transient-eddy standard deviation of the relative vorticity at $700 \mathrm{hPa}$. Contours are every $0.5 \times 10^{-5} \mathrm{~s}^{-1}$. Regions with values larger than $5 \times 10^{-5} \mathrm{~s}^{-1}$ are shaded. Correlation coefficients between BB on the left side and $\mathrm{LB}$ on the right side are $R=0.83$ for the entire domain; for ocean: $R=0.86$, land: $R=0.85$, flat land: $R=0.68$, and mountains: $R=0.89$. b (lower panels)

\subsubsection{The 700-hPa vorticity fields}

In order to investigate the downscaling ability away from surface forcings, we turn our attention to the 700$\mathrm{hPa}$ vorticity field. The relative vorticity of the $\mathrm{LB}$ at $700 \mathrm{hPa}$ shows a very good reproduction of the BB fields (Fig. 8a) over the ocean $(R=95 \%)$ and Rocky
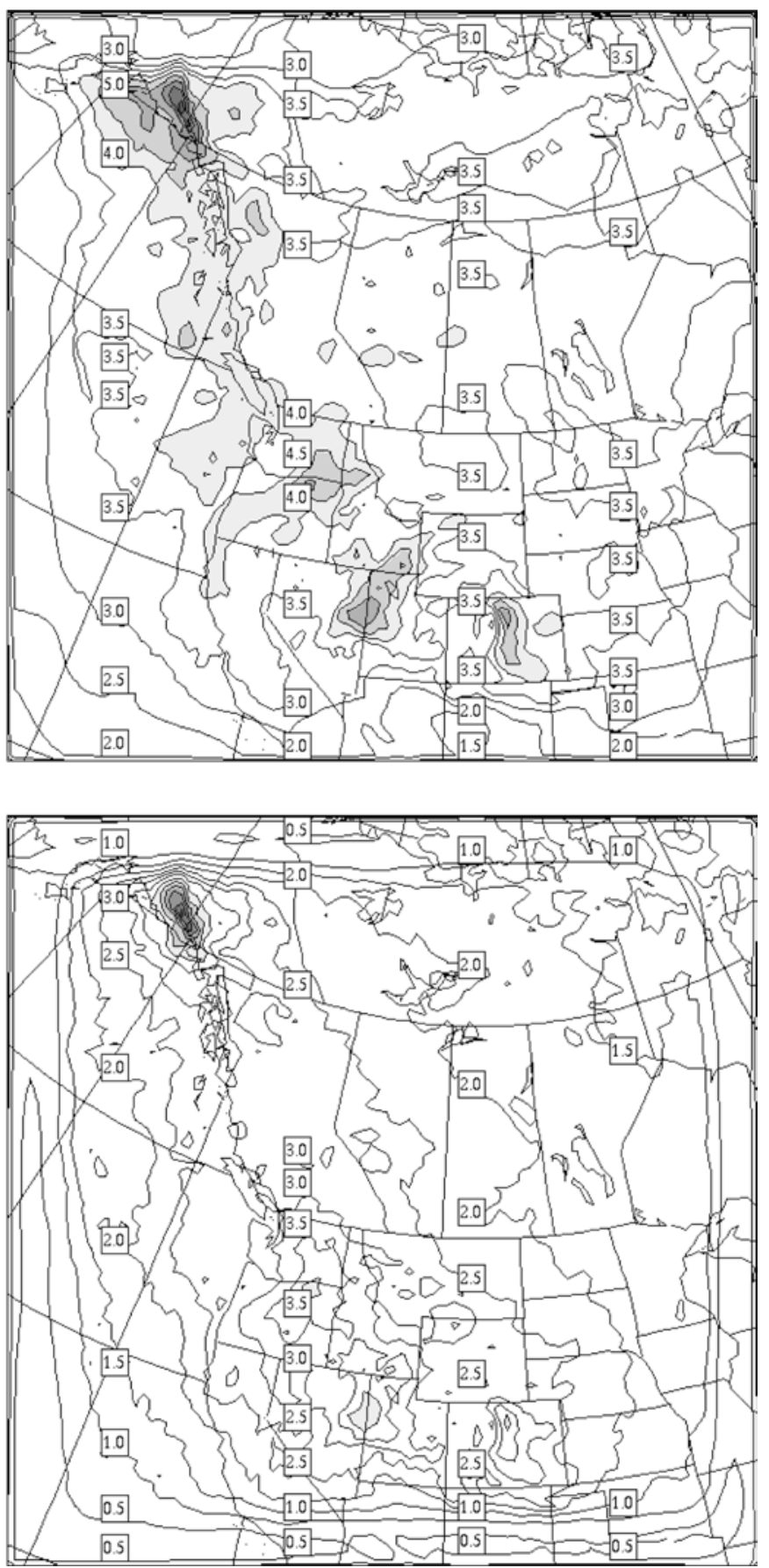

Transient-eddy standard deviation of the small-scale component of the relative vorticity at $700 \mathrm{hPa}$. Contours are every $0.5 \times 10^{-5} \mathrm{~s}^{-1}$ Correlation coefficients are $R=0.87$ for the entire domain; for ocean: $R=0.85$, land: $R=0.91$, flat land: $R=0.87$, and mountains: $R=0.94$ 
Mountains $(R=96 \%)$, but a noticeably smaller spatial correlation over the flat land region $(R=87 \%)$. The stationary small-scale component is well regenerated $(\Gamma$ $=102 \%)$, and correlates well over the Rocky Mountains $(R=91 \%)$ where strong surface forcing exists.

Transient-eddy standard deviation of the total and small scales is shown in Figs. 9a, 9b. These fields have the largest correlation over the Rocky Mountains $(R>89 \%)$. The spatial variability is underestimated for total field $\Gamma=82 \%$ and slightly over-estimated for small-scale component $\Gamma=109 \%$. It is important to mention again that the relaxation zone is excluded from the statistic calculations, but it is shown in the figure, as can be clearly seen on the right-hand side panel of Fig. $9 \mathrm{~b}$. The presence of the relaxation zone as such outstanding feature can be explained as follows: in this experiment the filtered wind that drives the Little Brother does not possess small scales. From the boundary towards the interior of the domain, the relaxation diminishes progressively, allowing the variability to develop accordingly, hence leaving an imprint of this variation near the boundaries. This effect can also be seen in the right-hand side panel of Fig. 7b. However, inhomogeneities in the horizontal distribution of precipitation make it less conspicuous.

Considering that the $700-\mathrm{hPa}$ surface is distant from surface forcings, it is noteworthy that the vorticity field still bears the signature of high mountains, which control circulations in this region. As it can be understood from Fig. 8a, mountain-induced disturbances are created when air is forced to rise over the Rocky Mountains, inducing upslope anticyclogenesis and lee cyclogenesis. Those features are well regenerated by the Little Brother (correlation is larger than $91 \%$ for means fields over the Rocky Mountains). Since small-scales that are absent in the lateral boundary conditions have to be generated by dynamical and physical processes in the free atmosphere, smaller correlation coefficients are expected compared with those of surface fields presented already. In the mean as well as in the transient vorticity fields, there is a noticeable decrease in correlation over the "flat land" region; this is probably due to domain size, which is not sufficiently large to allow the full development of small-scales features over the width of the domain (Jones et al. 1995).

These results show that the small-domain LB is successful in regenerating the features of the reference largedomain BB simulation. Several other fields where also inspected and showed the same results. Table 2 shows spatial correlation coefficient and ratio of spatial variance for screen-level specific humidity, screen-level temperature, geopotential and temperature at $700 \mathrm{hPa}$, and vertical velocity at $850 \mathrm{hPa}$. In this table we can see that stationary and transient components of total and small-scale fields have correlation coefficients larger than $94 \%$. Ratio of variance shows excellent regeneration of variability for the screen level fields $(\Gamma>95 \%)$, and somewhat weaker LB variability for fields further away from surface forcings $(\Gamma>87 \%)$.

These results are in agreement with those of Denis at al. (2002b, 2003). The fine-scale features, that are absent in the initial and lateral boundary conditions, are successfully regenerated by one-way nested LB $(R>94 \%)$, with exception of the relative vorticity at $700 \mathrm{hPa}$ that illustrates reduced correlation $(R>83 \%)$. It is useful to mention that the domain-size issue seems to be mainly responsible for reduced correlation for vorticity, despite the ability of reproduction for the other regional-climate features. Generally, better correlation was found over the Rocky Mountains region where stationary topographic and coastal forcings are important. Moreover, comparing with Denis et al. (2002b, 2003), the strong surface forcing improved the downscaling ability, especially for precipitation fields.

3.2 Sensitivity to the spatial resolution and temporal update frequency of lateral boundary conditions

In order to further evaluate the sensitivity of the oneway nested technique, different horizontal resolutions and update frequencies of lateral boundary conditions were used for experiments indicated in Table 1. The small-domain LB was nested in the filtered version of the high-resolution $\mathrm{BB}$; see filters $\mathrm{J} 1$, J6 and $\mathrm{J} 12$ with responses shown in Fig. 3. The J1 "filter" corresponds to

Table 2 Spatial correlation coefficients $(R)$ and ratio of variance $(\Gamma)$ between LB and BB simulations. The $\mathrm{R}$ and $\Gamma$ are calculated for: screen-level specific humidity, screen-level temperature, geopotential at 700-hPa, temperature at 700-hPa and vertical velocity at $850 \mathrm{hPa}$. The correlation coefficient and ratio of variance are presented for stationary components of all-scale and small-scale features and transient-eddy components of all-scale and small-scale features

\begin{tabular}{|c|c|c|c|c|c|c|c|c|c|c|}
\hline & \multicolumn{2}{|c|}{$\begin{array}{l}\text { Screen-level } \\
\text { specific } \\
\text { humidity }\end{array}$} & \multicolumn{2}{|c|}{$\begin{array}{l}\text { Screen-level } \\
\text { temperature }\end{array}$} & \multicolumn{2}{|c|}{$\begin{array}{l}\text { 700-hPa geopo- } \\
\text { tential }\end{array}$} & \multicolumn{2}{|c|}{$\begin{array}{l}700-\mathrm{hPa} \\
\text { perature }\end{array}$} & \multicolumn{2}{|c|}{$\begin{array}{l}850-\mathrm{hPa} \text { verti- } \\
\text { cal velocity }\end{array}$} \\
\hline & $R(\%)$ & $\Gamma(\%)$ & $R(\%)$ & $\Gamma(\%)$ & $R(\%)$ & $\Gamma(\%)$ & $R(\%)$ & $\Gamma(\%)$ & $R(\%)$ & $\Gamma(\%)$ \\
\hline Stationary components of total field & 99 & 100 & 99 & 100 & 99 & 100 & 99 & 98 & 99 & 97 \\
\hline Stationary components of small scales & 99 & 99 & 99 & 98 & 97 & 99 & 96 & 89 & 98 & 97 \\
\hline Transient eddy components of total field & 99 & 95 & 99 & 99 & 99 & 98 & 99 & 98 & 99 & 96 \\
\hline Transient eddy components of small scales & 98 & 97 & 98 & 100 & 94 & 87 & 97 & 94 & 98 & 93 \\
\hline
\end{tabular}


non-filtered $45 \sim \mathrm{km}$ grid $\mathrm{RCM}$ data and was also used to evaluate sensitivity to update frequencies of lateral boundary conditions. It is worth mentioning, however, that there is some filtering associated with $\mathrm{J} 1$ as the model variables are de-staggered, interpolated to pressure surfaces, re-interpolated to Gal-Chen coordinates and finally re-staggered. Experiments updated every 3, 6 or $12 \mathrm{~h}$ were labelled as $\mathrm{U} 8, \mathrm{U} 4$ and $\mathrm{U} 2$ respectively. Combination of update frequencies with different driving data resolution, as shown in Table 1, will give an
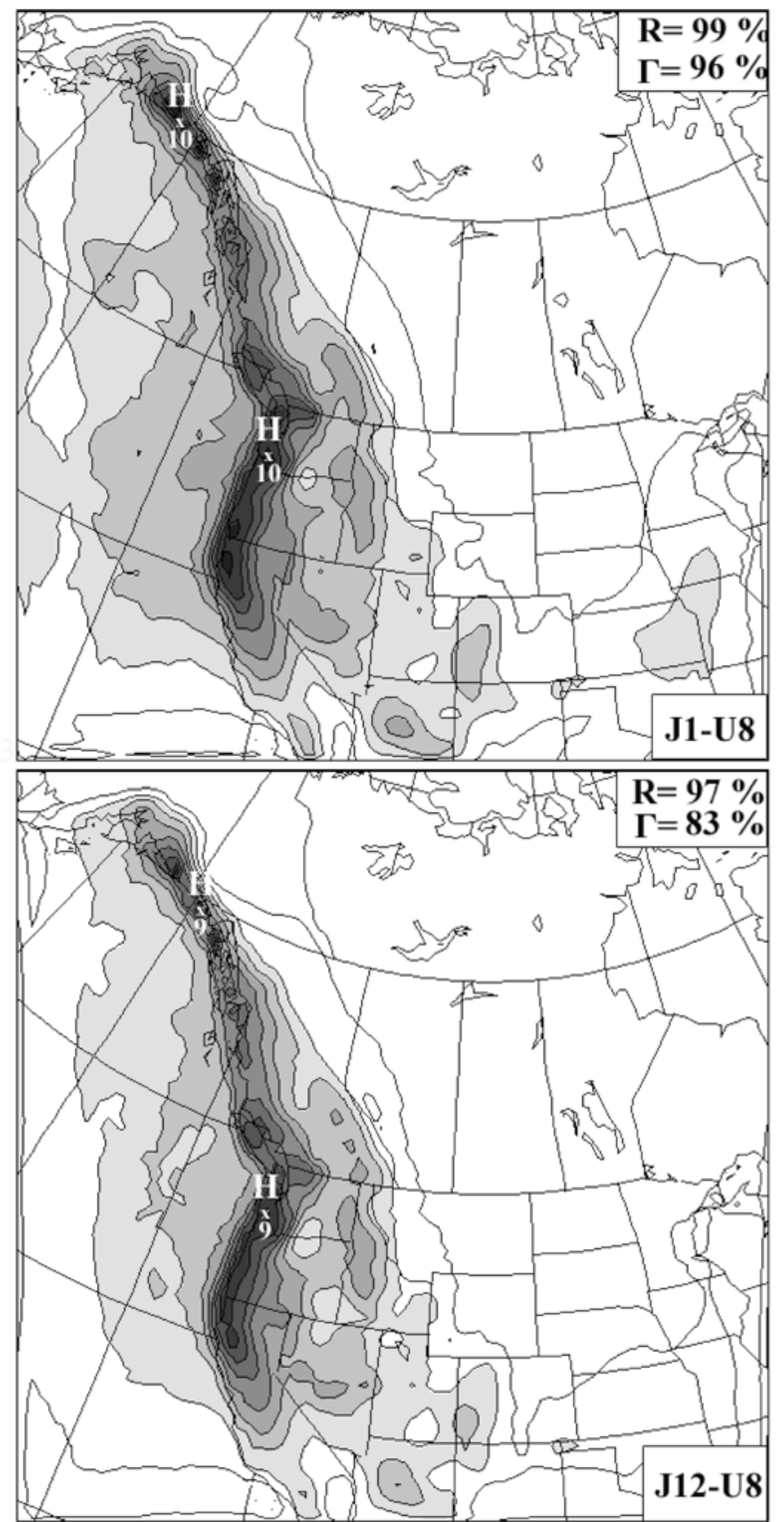

Fig. 10 Monthly mean precipitation rate. Contours are every $1 \mathrm{~mm} /$ day. Regions with precipitation larger than $2 \mathrm{~mm} /$ day are shaded. In the lower-right corners is indicated using combination of assessment of the sensitivity of regenerated small scales to lateral boundaries.

\subsubsection{Precipitation}

As mentioned, precipitation is generated by complex interactions between dynamics and parametrised physical processes in the RCM. The ability of the small-domain LB to regenerate the reference BB precipitation field, when nested with different lateral boundary con-
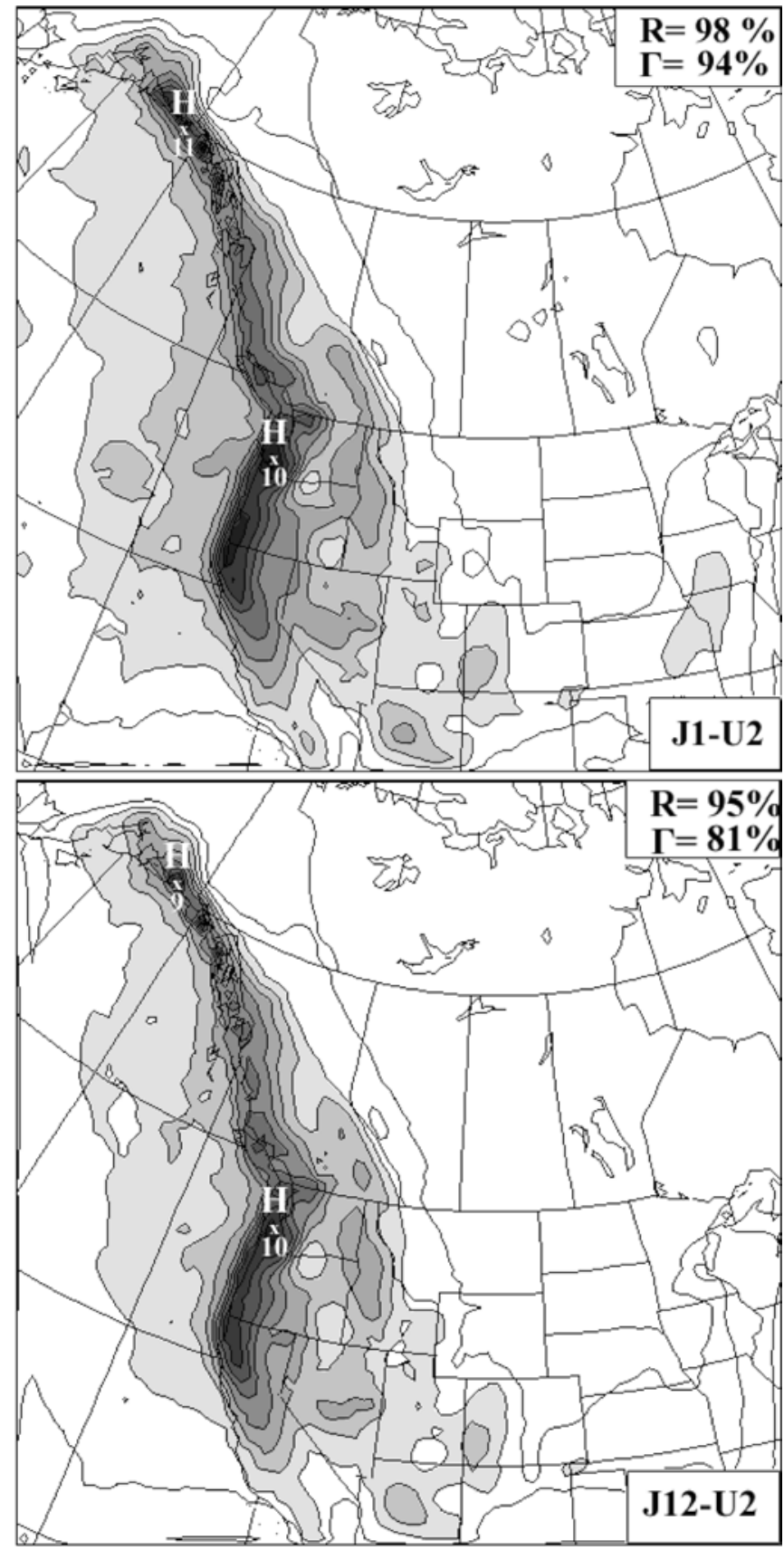

resolution of driving data $(J)$ and update frequency $(U)$. Correlation coefficients $(R)$ and variance ratios $(\Gamma)$ between the LB and BB are indicated in the upper-right corners 
ditions, gives an estimation of LB sensitivity. As objective evaluators, spatial correlation coefficient and ratio of spatial variance between $\mathrm{LB}$ and reference $\mathrm{BB}$, are calculated for J1-U8, J1-U2, J12-U8 and J12-U2 combination of driving data horizontal resolution $(\mathrm{J})$ and update frequency (U).

Figure 10 shows monthly mean precipitation fields averaged over four February simulations of LB. The stationary component of precipitation is regenerated

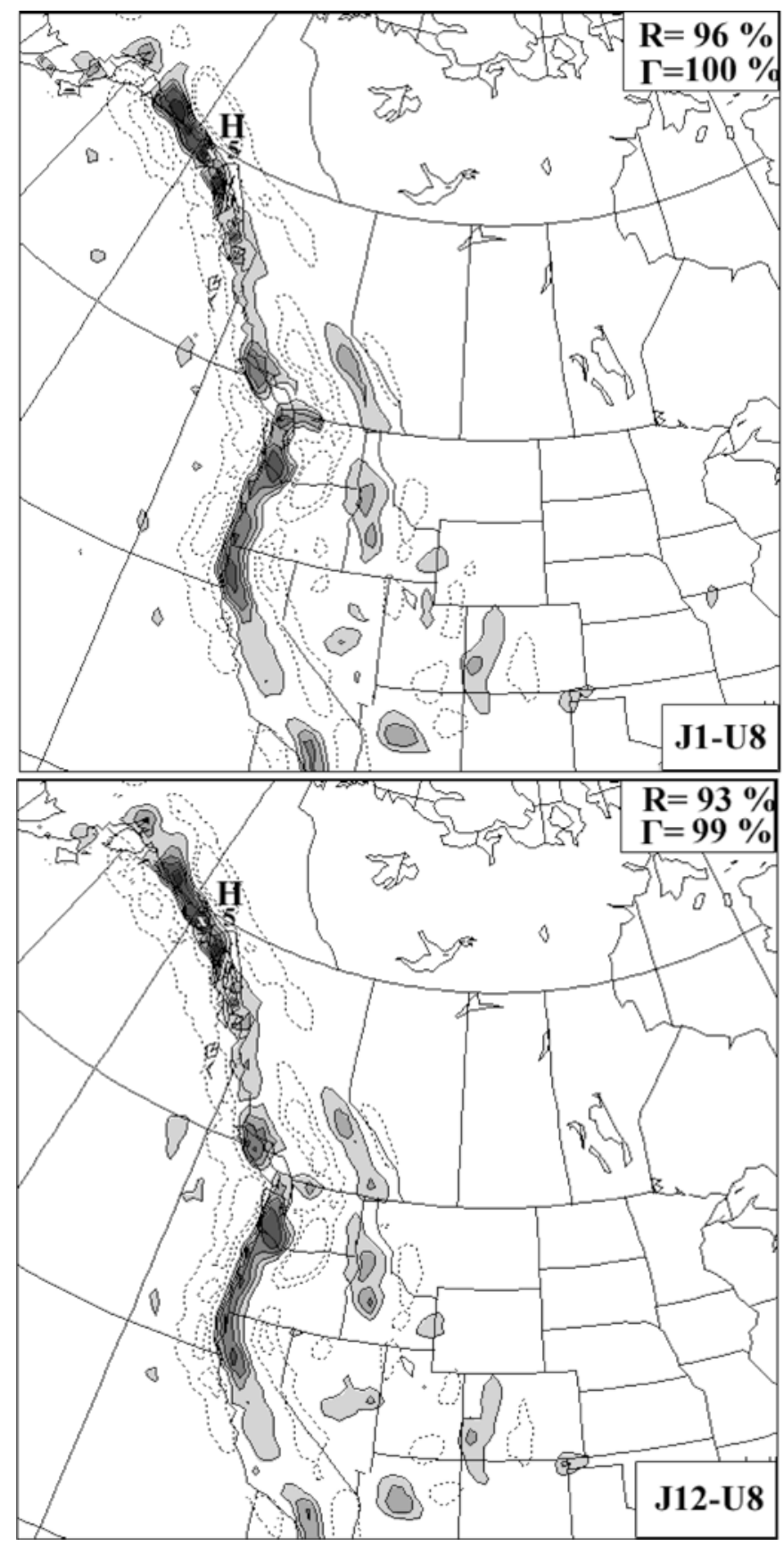

Fig. 11 Stationary small-scale component of the precipitation rate. Contours are $\{-5.0,-3.0,-1.0,-0.5,0.5,1,1.5,2,3,5\} \mathrm{mm} /$ day. Shaded regions have values larger than $0.5 \mathrm{~mm} /$ day. In the lowerright corners is indicated used combination of resolution of driving well in all cases, and spatial correlation coefficients are larger than $95 \%$ for all four $\mathrm{J}-\mathrm{U}$ combinations. No noticeable changes were found in pattern positions nor intensities when resolution and/or update frequency of the driving data were changed. The only remarkable difference is in the precipitation over the nesting zone, which is the best simulated with LB nested in non-filtered data (J1) that include small-scales in the LBC. Spatial variability of precipitation rate is underestimated

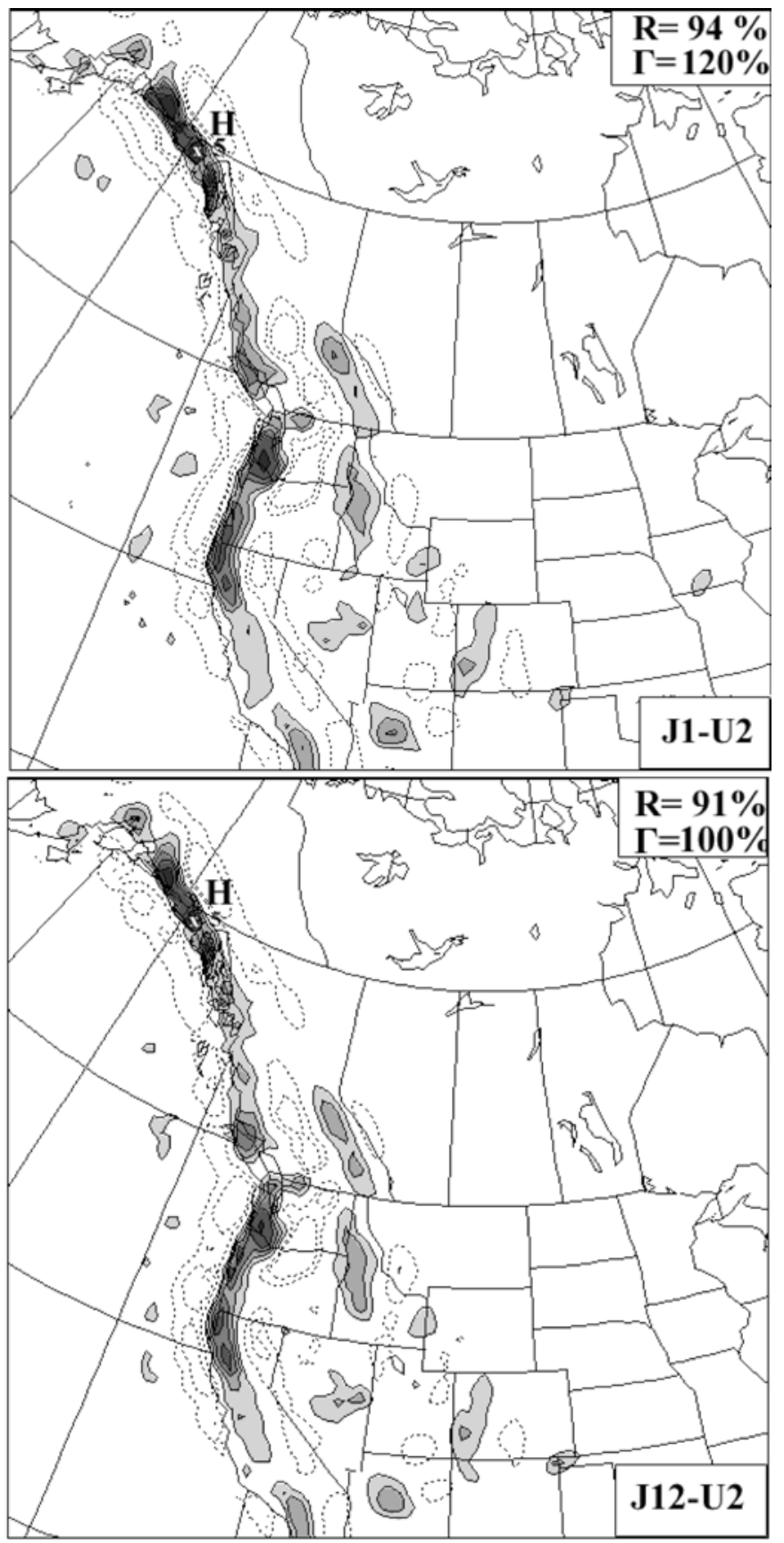

data $(J)$ and update frequency $(U)$. Correlation coefficients $(R)$ and variance ratios $(\Gamma)$ between the $\mathrm{LB}$ and $\mathrm{BB}$ are indicated in the upper-right corner 

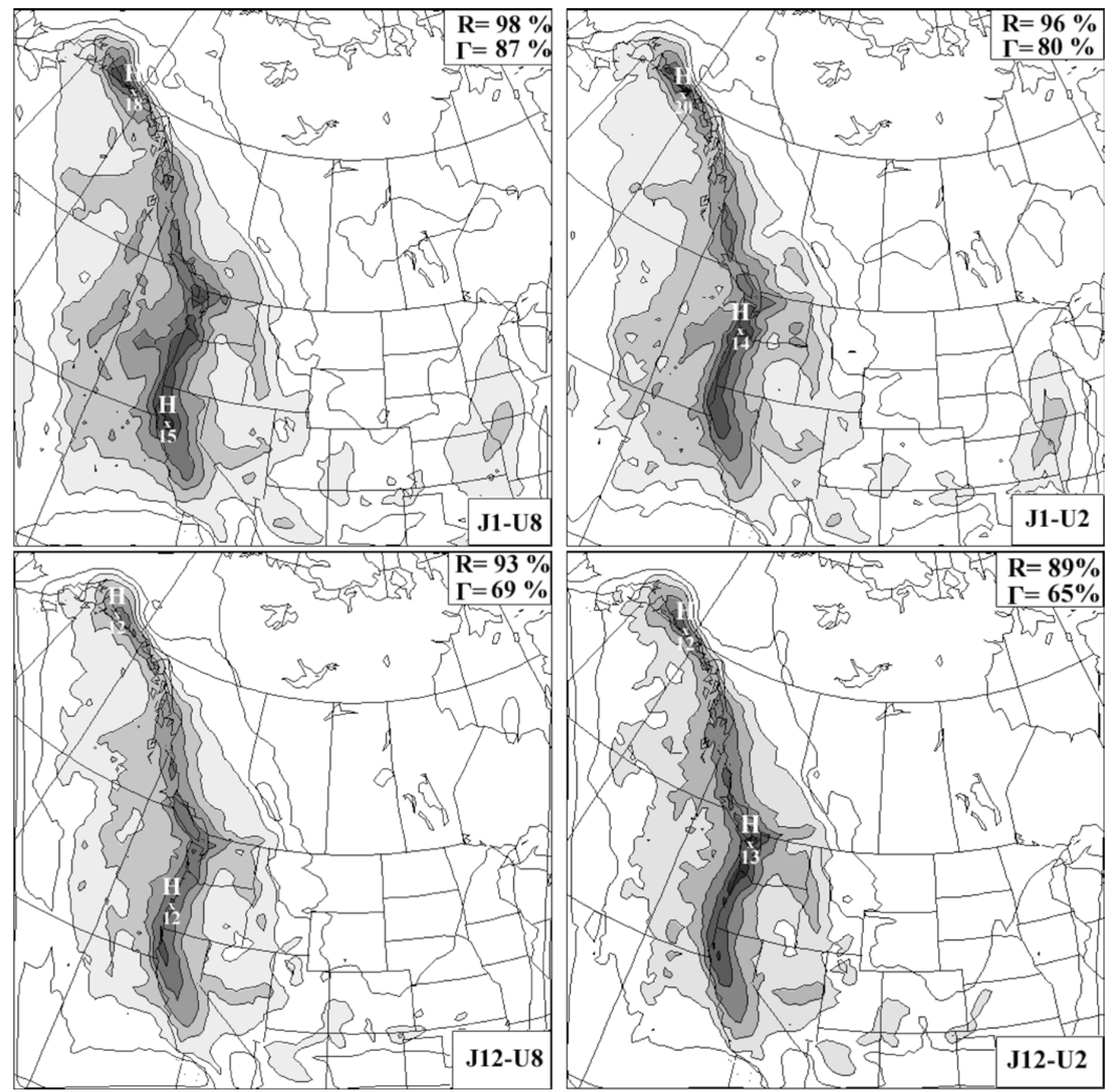

Fig. 12 Transient-eddy standard deviation of precipitation rate. Contours are every $2 \mathrm{~mm} /$ day. Shaded regions have values larger than $4 \mathrm{~mm} /$ day. In the lower-right corners is indicated used

in all cases but it remains larger than $81 \%$. Underestimation of precipitation maximum and variance is significant when resolution is changed from $\mathrm{J} 1$ to $\mathrm{J} 12$ ( $\Gamma$ drops from $96 \%$ to $83 \%$ ), but it seems insignificant when update frequency is decreased from eight to two updates per day ( $\Gamma$ only drops from $96 \%$ to $94 \%$ ). Figure 11 shows the stationary small-scale component of precipitation fields. The important surface forcing present in our computational domain seems to be responsible for the successful regeneration of small-scale

combination of resolution of driving data $(J)$ and update frequency $(U)$. Correlation coefficients $(R)$ and variance ratios $(I)$ between the $\mathrm{LB}$ and $\mathrm{BB}$ are indicated in the upper-right corner

BB precipitation near mountains. Similar pattern positions and correlation coefficient larger than $91 \%$ in combination with a well-simulated variance suggest that small-scale precipitation is very well regenerated.

Temporal variability of the total precipitation field and its small-scale component are respectively presented in Figs. 12 and 13. Comparison of resolution jump J12 against $\mathrm{J} 1$ shows similar pattern positions, a small decrease in correlation coefficients from $98 \%$ to $93 \%$ but a substantial reduction in small-scale transient-eddy vari- 

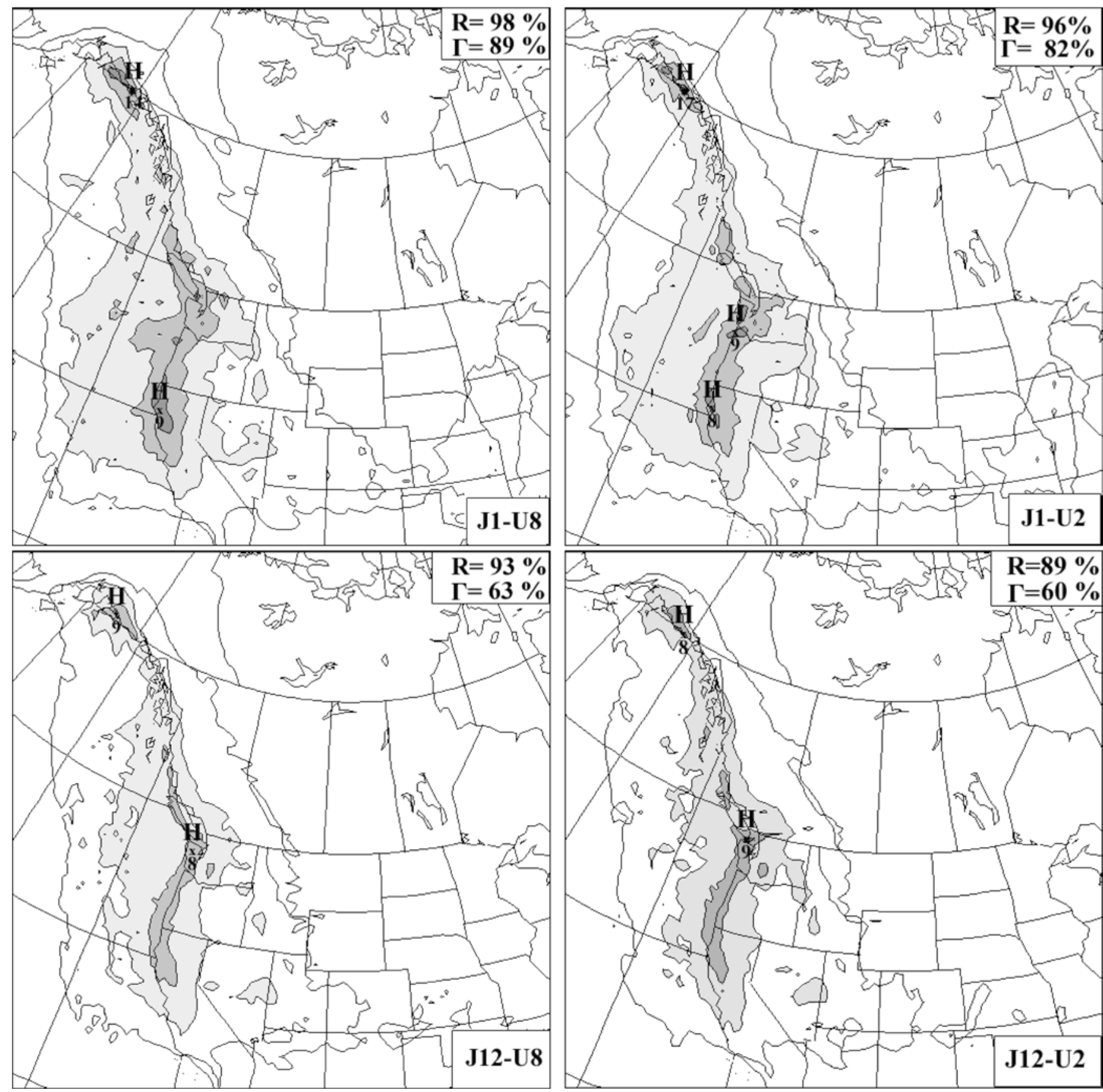

Fig. 13 Transient-eddy standard deviation of small-scale component of precipitation rate. Contours are every $2 \mathrm{~mm} /$ day. Shaded regions have values larger than $4 \mathrm{~mm} /$ day. In the lower-right corners is indicated used combination of resolution of driving data

ability, from $89 \%$ to $63 \%$ for J12-U8. A more modest decrease of small-scale transient-eddy variability is due to increasing update interval, from $89 \%$ to $82 \%$ for $\mathrm{J} 1$ case. This variance reduction is a direct consequence of the reduced amplitude of transient synoptic-scales phenomena.

The pattern position and intensity, as could be seen in Figs. 10-13, are similar in the four experiments performed. Moreover, the results show good regeneration
$(J)$ and update frequency $(U)$. Correlation coefficients $(R)$ and variance ratios $(\Gamma)$ between the $\mathrm{LB}$ and $\mathrm{BB}$ are indicated in the upper-right corner

of the small-scale and total stationary precipitation fields; the worst simulation is, as expected, J12-U2 with correlation coefficient still greater than $91 \%$ and variance ratios of $81 \%$ and $100 \%$, for small-scale and total stationary eddies, respectively. Transient fields for the same J12-U2 simulation have correlations of $89 \%$ but rather small variance ratios reaching values of around $60 \%$. Concerning the downscaling ability, the sensitivity of the precipitation seems to indicate that an operational 
Fig. 14 Summary diagrams showing the effect of different horizontal resolution jumps. In these experiments lateral boundary conditions are updated eight and two times per day, on the left and the right side, respectively. The stationary component of sea-level pressure, precipitation rate, screen-level temperature and $500-\mathrm{hPa}$ vorticity are presented. Circles: J1, triangles: J6 and squares: J12. Open symbols are for total fields and shaded symbols are for small-scale field
U8 (3 hour update-frequency)
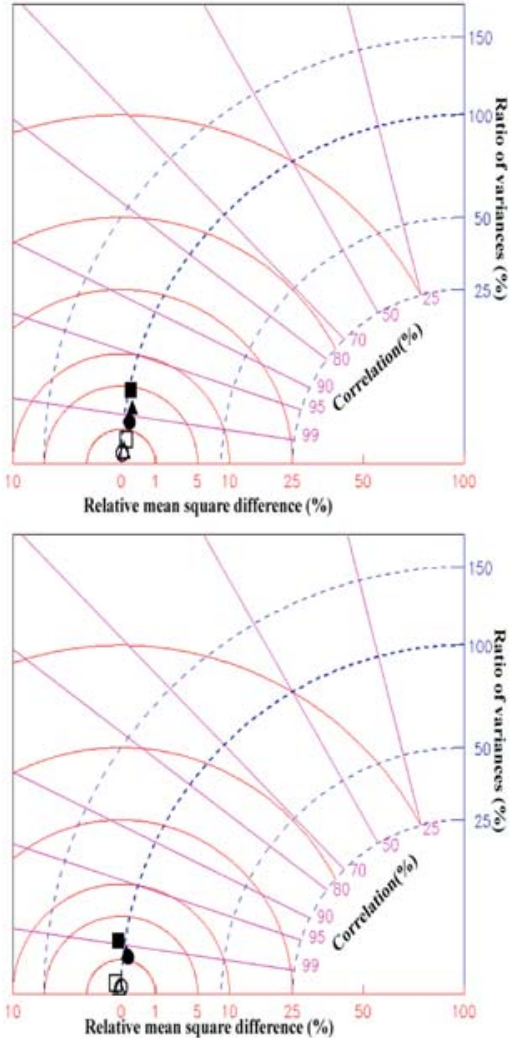

\section{Screen-level} temperature sea-level pressure

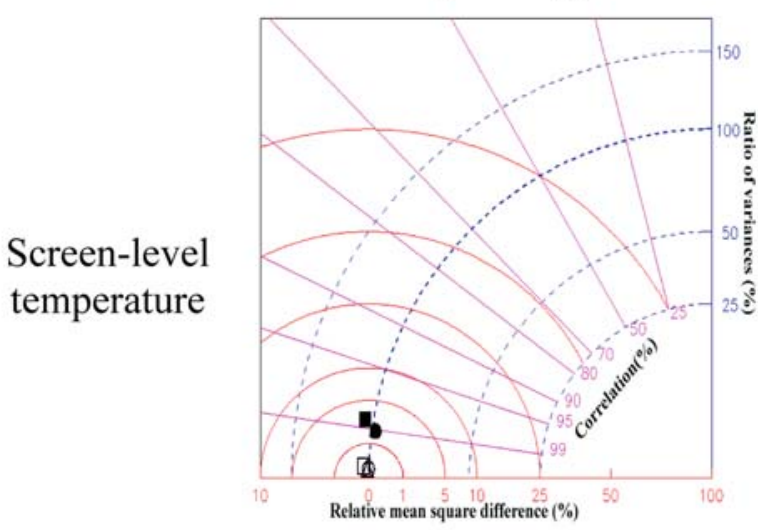

U2 (12 hour update-frequency)
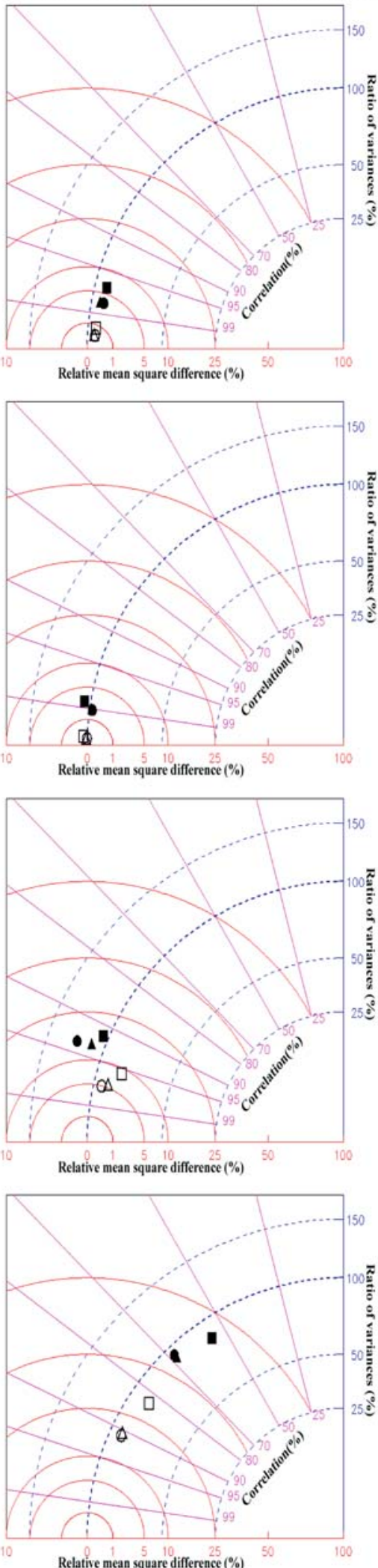

climate-applicability of the one-way nesting technique requires $\mathbf{J}<12$ and $\mathrm{U}>2$ for a $45 \sim \mathrm{km}$ grid-point RCMs. It is important to mention that, the evaluation is per- formed for a perfect-model approach and it takes in consideration only errors generated by the one-way nested technique. 
Fig. 15 As in Fig. 14 but for transient component of the fields
U8 (3 hour update-frequency)
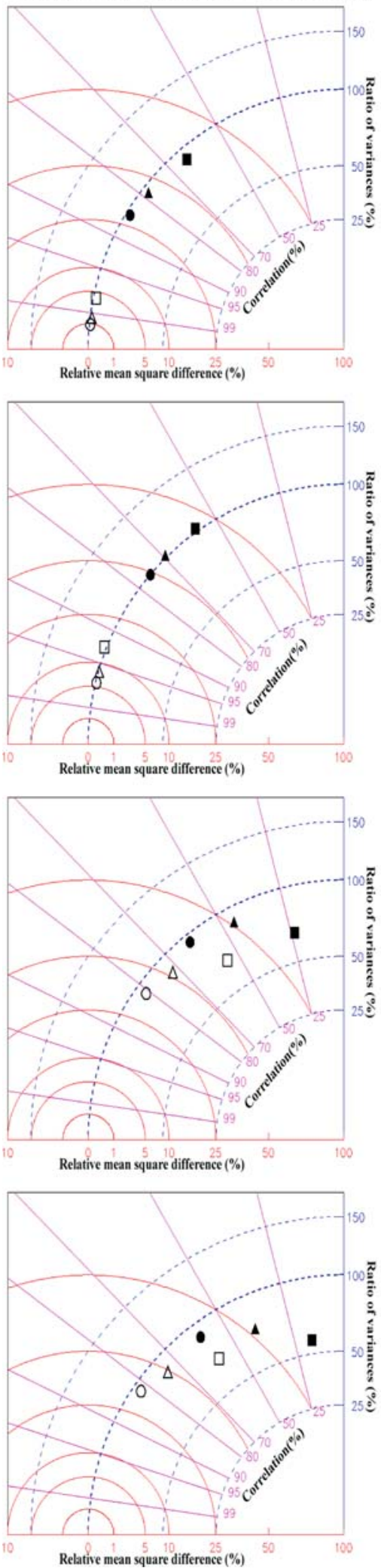

U2 (12 hour update-frequency)
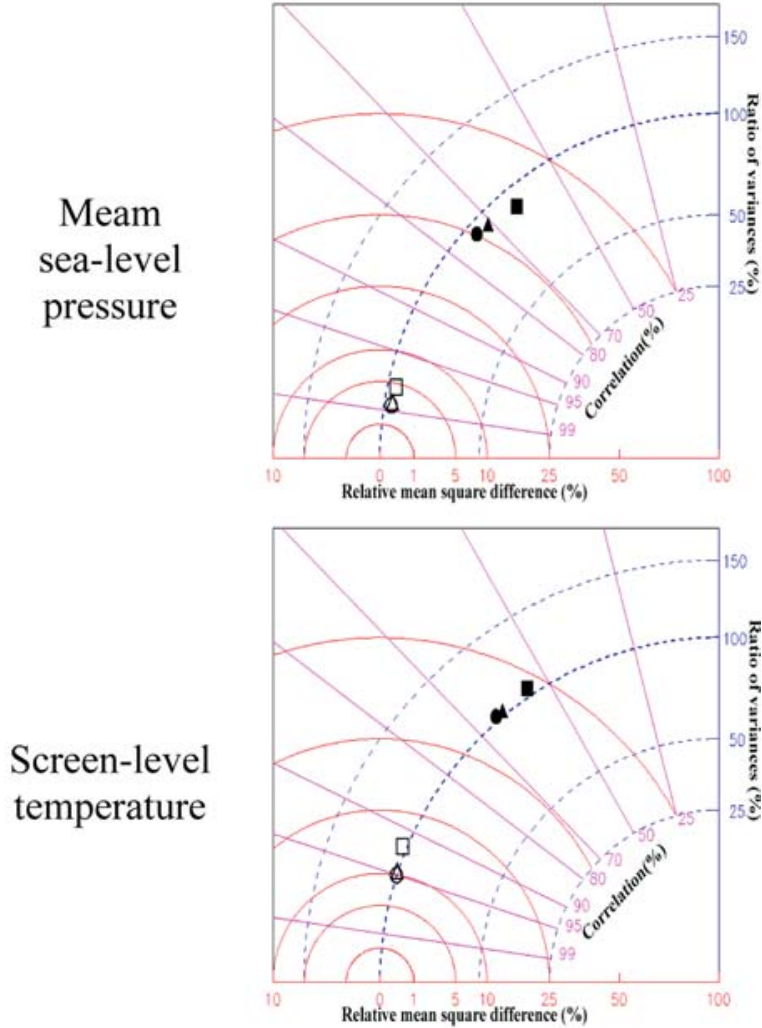

Meam sea-level pressure
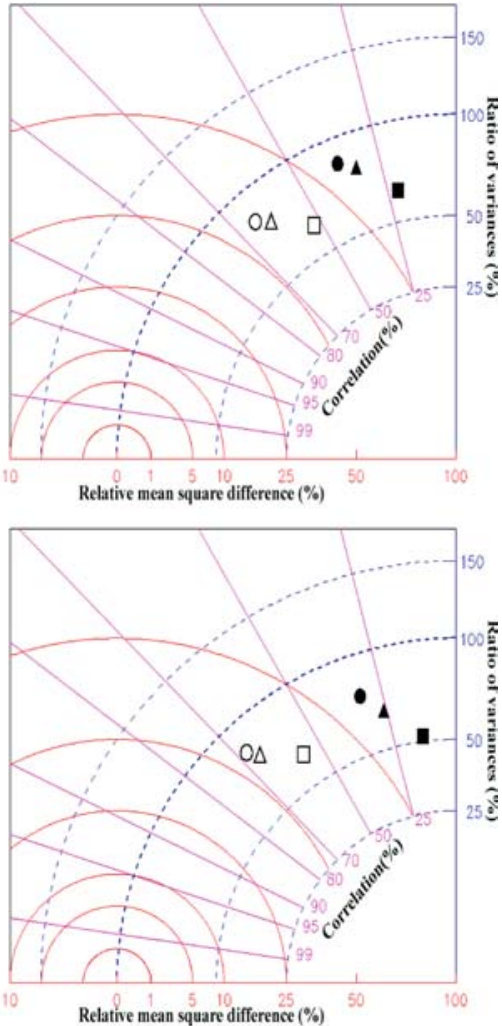

As noted, the ratios of variance of the stationary and transient precipitation fields for different nesting conditions show more sensitivity to an increase in spatial resolution than in update frequency. Minor differences are noted for the stationary small-scale precipitation field that is very well regenerated by LB ( $\Gamma$ between $99 \%$ 
and $120 \%$ ); this might indicate important topographical forcing influences. These results lead us to believe that a one-way nested RCM is more sensitive to decrease in spatial resolution of lateral boundary conditions from $\mathrm{J} 1$ to $\mathrm{J} 12$ than to a change of update interval from $\mathrm{U} 8$ to U2. This finding agrees with that of Denis et al. (2003).

\subsubsection{Summary diagrams}

The comparison of RCM fields with different nesting resolution in space and time is depicted using summary diagrams proposed by Taylor (2001). For this purpose, correlation coefficient, relative mean square difference and ratio of variance between two fields are all displayed on a single plot. This diagram is explained in detail in Taylor (2001) and Denis et al. (2003). Relative mean difference is presented as radial distance from the origin of the abscissa, the standard deviation ratio is shown as the radial distance from lower-right corner and the correlation coefficient as the azimuthal angle.

As stated in previous discussions, spatial and temporal decomposition of the fields have been employed. Total and small-scale components are presented in the same graph, either for stationary or transient components. In the summary diagrams for the stationary field, spatial correlation coefficient $R$, ratio of spatial variance $\left(\sigma \frac{* 2}{L B} / \sigma \frac{* 2}{B B}\right)$ and normalised spatial mean square difference $\left(d \frac{* 2}{L B L B} / \sigma \frac{* 2}{B B}\right)$ between Little and Big Brothers are presented. For the transient component of the fields, a temporal correlation coefficient, a ratio of spatially averaged temporal variance $\left(\left\langle\sigma_{L B}^{2 \prime}\right\rangle /\left\langle\sigma_{B B}^{2 \prime}\right\rangle\right)$ and temporal mean square difference $\left(\left\langle\overline{d_{L B L B}^{2 \prime}}\right\rangle /\left\langle\sigma_{B B}^{\prime 2}\right\rangle\right)$ are presented (for more details, see Denis et al. 2003). This type of temporal comparison for the transient part is usually not attempted for global climate models since two simulations become independent after a few weeks, and therefore the temporal correlation vanishes. In the case of nested RCMs, however, the control exerted by the lateral boundary nesting forces the solution to maintain certain resemblance (e.g. correlation) with the driving data (de Elía et al., 2002). The degree of the control exerted by LBC is in itself an interesting question.

Figure 14 shows results concerning the sensitivity of one-way nested technique to spatial resolution of driving data in two cases: updated every $3 \mathrm{~h}$ (left-hand column) and $12 \mathrm{~h}$ (right-hand column). Stationary fields of sealevel pressure and screen temperature show weak sensitivity to resolution jump; patterns of total field and small-scale component are simulated with high correlation $(\mathrm{R}>97 \%)$ and spatial variability was successful reproduced. The stationary precipitation field shows a large range of variance ratio $(80 \%$ to $120 \%)$ and a weaker correlation but that remains larger than $90 \%$. Furthermore, the mean square difference is augmented when resolution jumps increase from J6 to J12. Important differences are noted between J6 and J12 for 500$\mathrm{hPa}$ relative vorticity field. For all stationary fields, excluding the $500-\mathrm{hPa}$ vorticity, change in update frequency did not cause an important change in sensitivity. It is also worth noting that significant differences were not found in simulated fields between driving resolutions $\mathrm{J} 1$ and $\mathrm{J} 6$.

The transient component of fields is presented in Fig. 15. Temporal correlation is much more demanding than the spatial correlation since it measures the ability of the model to regenerate perturbation at the appropriate time and place and, as expected, results show smaller correlation values for transients (see Fig. 15) than for stationary (Fig. 14). For climate application, it is more important to reproduce properly the patterns of variability rather than the correct timing of events. The transient component of sea-level pressure and screen temperature is well simulated $(R>91 \%)$. A decrease in correlation of small-scale components is noticeable, but it remains above $55 \%$. A reduced transient-eddy variance is clearly seen for precipitation and $500-\mathrm{hPa}$ vorticity field. Difference between 6- and 12-h updated fields is not apparent for mean slp and screen-level temperature fields, but the two other fields presented show important reduction in correlation. As for the stationary component, increasing the resolution of the driving data from $\mathrm{J} 12$ to $\mathrm{J} 6$ has an evident positive influence, but increasing it further from $\mathrm{J} 6$ to $\mathrm{J} 1$ produces little improvement.

Additional information regarding sensitivity of oneway nesting to spatial resolution of driving data can be obtained by comparing the results shown above for the region of western North America against those presented in Figs. 16 and of Denis et al. (2003) for the region of eastern North America. Comparison of the stationary small-scale precipitation field shows that it is significantly affected by difference in surface forcing; for example, the complex topographic forcings over the western America improved correlation from $65 \%$ to $97 \%$ for the J1 experiment. The LB ability to regenerate transient small-scale sea level pressure fields is improved from $75 \%$ to $83 \%$, and slightly deteriorated for the stationary total $500-\mathrm{hPa}$ relative vorticity field (from $99 \%$ to $95 \%$ ). Concerning the impact of different resolution jump, it is noteworthy that changes in driving data resolution from $\mathrm{J} 12$ to J6 have improved the correlation coefficient more than a change from $\mathrm{J} 6$ to $\mathrm{J} 1$. This characteristic is in accord with the findings of Denis et al. (2003); it is another evidence of an important improvement that can be anticipated by increasing the driving data resolution from $\mathrm{J} 12$ to $\mathrm{J} 6$.

The use of summary diagrams to summarise results has been helpful to visualise the improvement gained in increasing the spatial resolution and update frequency of lateral boundary conditions. The Big-Brother approach presented is idealised and the results presented must be considered as an evaluation of sensitivity of the one-way nested technique. As was seen, the resolution jump that corresponds to filter $\mathrm{J} 12$ was successful in reproducing fields exhibiting large-scale behaviours, but for complex fields such as precipitation or vorticity, the capability of 
LB to reproduce the reference $\mathrm{BB}$ was more limited. The update frequency has distinctive influence on RCM nested with $\mathrm{J} 1$ resolution and there is a noticeable difference between two and four updates per day. The influence of update frequency on $\mathrm{J} 12$ resolution of driving data is limited. Considering all presented advantages and limitations to the increase of spatial resolution of driving data and update frequency, the same conclusion as in Denis et al. (2003) could be indicated: the J12-U2 combination is the "minimal" configuration for an acceptable reproduction of reference BB fields, but better results are obtained with the setup J6-U4.

\section{Conclusions}

The primary aim of this study was to investigate the downscaling ability of a one-way nested RCM over complex terrain, and its sensitivity to the spatial resolution as well as to the temporal update frequency of the lateral boundary conditions. In order to complement the study of Denis et al. (2002b, 2003) who analysed the behaviour of a nested the model on the East Coast of North America, this study focused over the west coast of North America, in the region where the Rocky Mountains exert a strong influence on the regional climate.

Results from a perfect-model approach nick named the Big-Brother Experiment designed to investigate errors associated with downscaling technique. This approach consists of primary establishing a virtualreality Big-Brother climate in a large area of approximately $8900 \mathrm{~km}^{2}$ using the $45-\mathrm{km}$ grid-mesh Canadian RCM nested with NCEP analyses. The resulting data are filtered and used as "perfect" initial and lateral boundary conditions for the same regional model, but integrated over a smaller sub-area of approx. $4500 \mathrm{~km}^{2}$ the Little-Brother simulations. Results obtained by comparing climate statistics for four winter months of the Little Brother to the Big Brother over the same area, point us to the following conclusions:

1. The mean sea level pressure, precipitation, geopotential and temperature at $700 \sim \mathrm{hPa}$ and screen-level fields were successfully regenerated by the one-way nested RCM. The downscaling ability of the Little Brother is also significant for transient eddies, as well as for the stationary components.

2. The precipitation is strongly modulated by the Rocky Mountains and coastlines. The best correlation between Big and Little Brother is found over the Rocky Mountains region. Complex topography enhances the downscaling ability of precipitation compared to the results of Denis et al. (2003) obtained over the east coast of North America.

3. The Little-Brother 700-hPa vorticity field showed the influence of the high mountain forcing, by the existence of anticyclogenesis on the windward side and lee cyclogenesis in the other.
4. The downscaling ability found in various fields showed the climate applicability of the one-way nesting technique up to T30-12 h combination for 45$\mathrm{km}$ grid-mash RCM nested in a perfect environment, in agreement with the results of Denis et al. (2003).

5. Change in spatial resolution of driving data from T30 to T60 has more repercussion on the downscaling ability than a change from T60 to T360; this is more pronounced in the transient than in the stationary component of the fields. Important improvements can be anticipated by increasing of driving data resolution from $\mathrm{T} 30$ to $\mathrm{T} 60$.

6. Update frequency of four times a day (every $6 \mathrm{~h}$ ) showed better climate regeneration than that of twice a day (every $12 \mathrm{~h}$ ) for a resolution of T360 of the nesting data. As more scales were filtered from initial and lateral boundary conditions, results became less sensitive to variations of the update frequency. Little improvement was found for resolution T30 with change of 12 to $3 \mathrm{~h}$.

7. Finally, the T30-12 h combination can adequately reproduce the reference Big-Brother fields, but better results can be obtained with the setup T60-6 h.

As reported by Anthes et al. (1985) and subsequent studies (e.g. Vukicevic and Errico 1990; Peagle et al. 1997; Laprise et al. 2000; de Elía et al. 2002), predictability in nested limited-area models (LAM) is different from global models. Errors in the initial conditions do not grow beyond an asymptotic value that is much smaller than the value expected for a total loss of predictability as in a global model. Our results of the correlation coefficients for the transient components are a good indicator of the degree of control exerted by the lateral boundary nesting. Significant temporal correlation $(R>92 \%)$ is found for total sea-level pressure and screen temperature fields, and moderate $(R>50 \%)$ for precipitation and $500-\mathrm{hPa}$ relative vorticity fields (see Figs. 15). As expected, a smaller degree of control is established for small-scale phenomena that are not directly controlled by nesting; correlation larger than $55 \%$ for slp and screen-level temperature and weakly correlated small scales $(R \sim 25 \%)$ for precipitation and vorticity fields. Considering all this and the knowledge that a climate model is skillful if it correctly reproduces the statistical characteristics over some period, the weak time correlation for some variables does not bear any negative consequences on the skill of RCM for climate applications. Moreover, the predictability presented is significantly larger than zero, unlike that for global models, the reason being that RCM have fewer degrees of freedom than global models due to the imposed circulation at their lateral boundaries by nesting data.

It is important to bear in mind that all experiments in this work were based on a perfect-model approach, and therefore the conclusions that can be derived should be interpreted cautiously regarding the evaluation of sensitivity for the one-way nested technique. Deficiencies in the nesting model and nesting data may require different 
horizontal resolution jumps and/or update frequencies for an adequate regional climate modelling system.

Acknowledgements This work was funded by the Canadian Foundation for Climate and Atmospheric Sciences (CFCAS) and the Consortium "Ouranos" for regional climate and climatechange impact studies. We are also greatful to the regional climate modelling staff at UQAM for their valuable technical help with the Canadian RCM, and to Claude Desrocher for maintaining a userfriendly computing environment.

\section{References}

Ahmed N, Natarajan T, Rao KR (1974) Discrete cosine transformation. IEE Trans Computers C-23: 90-93

Anthes RA, Kuo YH, Baumhefner DP, Errico RM, Bettge TW (1985) Predictability of mesoscale motions. Advance in Geophysics vol. 288, Academic Press, New York, pp 159-202

Bergeron G, Laprise R, Caya D (1994) Formulation of the Mesoscale Compressible Community (MC2) model., Cooperative Centre for Research in Mesometeorology, pp 165 (Available from Prof. R. Laprise, Département des Sciences de la Terre et de l'Atmosphére, Universitédu Québec áMontréal, C.P. 8888, Succursale "Centre-Ville," Montréal, PQ, H3C 3P8, Canada)

Biner S, Caya D, Laprise R, Spacek L (2000) Nesting of RCMs by imposing large scales. In: Research activities in Atmospheric and Oceanic Modelling, WMO/TD - 987, Rep 30: 7.3-7.4

CAS/JSC WGNE (1999) Report of Fourteenth Session of the CAS/ JSC Working Group on Numerical Experimentation (Recherche en Prévision Numérique, Environnement Canada, Doval, Québec, Canada, 2-6 November 1998). 14, WMO/TD- 964, World Meteorolgical Organisation pp 28

CAS/JSC WGNE (2000) Report of Fifteenth Session of the CAS/ JSC Working Group on Numerical Experimentation (Naval Research Laboratory, Monterey, CA, USA, 25-29 October 1999). 15, WMO/TD- 1024, World Meteorological Organisation pp 29

Caya D, Laprise R (1999) A semi-implicit semi-Lagrangian regional climate model: the Canadian RCM. Mon Weather Rev 127: $341-362$

Davies HC (1976) A lateral boundary formulation for multi-level prediction models. Q J R Meteorol Soc 102: 405-418

de Elía R, Laprise R, Denis B (2002) Forecasting skill limits of nested, limited-area models: a perfect-model. approach. Mon Weather Rev 130: 2006-2023

Denis B, CotéJ, Laprise R (2002a) Spectral decomposition of twodimensional atmospheric fields on limited-area domains using the discrete cosine transform (DCT). Mon Weather Rev 130: $1812-1829$

Denis B, Laprise R, Caya D, Cote J (2002b) Downscaling ability of one-way nested regional climate model: the Big-Brother Experiment. Clim Dyn 18: 627-646

Denis B, Laprise R, Caya D (2003) Sensitivity of a regional climate model to the spatial resolution and temporal updating frequency of lateral boubdary conditions. Clim Dyn 20: 107-126

Dickinson RE, Errico RM, Giorgi F, Bates GT (1989) A regional climate model for the western United States. Clim Change 15: $383-422$
Giorgi F, Bates GT (1989) On the climatological skill of a regional model over complex terrain. Mon Weather Rev 117: 2325-2347

Giorgi F, Mearns LO (1999) Introduction to special section: regional climate modeling revisited. J Geophys Res 104: 63356352

Hong S-Y, Leetmaa A (1999) An evaluation of the NCEP RSM for regional climate modeling. J Clim 12: 592-609

Jones RG, Murphy JM, Noguer M (1995) Simulation of climate change over Europe using a nested regional-climate model. I: assessment of control climate, including sensitivity to location of lateral boundaries. Q J R Meteorol Soc 121: 1413-1449

Jones RG, Murphy JM, Noguer M, Keen AB (1997) Simulation of climate change over Europe using a nested regional-climate model. II: comparison of driving and regional model responses to a doubling of carbon dioxide. Q J R Meteorol Soc 123: 265292

Kain JS, Fritsch JM (1990) A one-dimensional entraining/detraining plume model and its application in convective parameterization. J Atmos Sci 47: 2784-2802

Laprise R, Caya D, Bergeron G, Giguére M (1997) The formulation of AndréRobert MC2 (Mesoscale Compressible Community) model. In: Lin C, Laprise R, Ritchie H (eds) The André J. Robert Memorial Volume, companion volume to Atmos-Ocean 35: 195-220

Laprise R, Caya D, Giguére M, Bergeron G, CotéH, Blanchet J-P, Boer G, McFarlane AN (1998) Climate and climate change in western Canada as simulated by the Canadian regional climate model. Atmos-Ocean 36: 119-167

Laprise R, Ravi Varma MK, Denis B, Caya D, Zawadzki I (2000) Predictability in a nested limited-area model. Mon Weather Rev 128: 4149-4154

Leung LR, Ghan SJ (1999) Pacific Northwest climate sensitivity simulated by a regional climate model driven by a GCM. Part I: control simulations. J Clim 12: 2010-2030

Marinucci MR, Giorgi F (1992) A $2 \mathrm{XCO}_{2}$ climate change scenario over Europe generated using a limited area model nested in a general circulation model. I: present-day climate simulation. J Geophys Res 97: 9989-10009

Peagle J, Yang Q, Wang M (1997) Predictability in limited area and global models. Meteorol Atmos Phys 63: 53-69

Robert A, Yakimiw E (1986) Identification and elimination of an inflow boundary computational solution in limited area model integrations. Atmos-Ocean 24: 369-385

Takle ES, Gutowski WJ, Jr., Arritt RW, Pan Z, Anderson CJ, da Silva RR, Caya D, Chen S-C, Giorgi F, Christensen JH, Hong S-Y, Juang H-MH, Katzfey J, Lapenta WM, Laprise R, Liston GE, Lopez P, McGregor J, Pielke RA Sr, Roads JO (1999) Project to Intercompare Regional Climate Simulations (PIRCS): description and initial results. J Geophys Res 104: 19,44319,461

Taylor KE (2001) Summarizing multiple aspects of model performance in a single diagram. J Geophys Res 106: 7183-7192

Vukucevic T, Errico R (1990) The influence of artificial and physical factors upon predictability estimates using a complex limited-area model. Mon Weather Rev 118: 1460-1482

Warner TT, Peterson RA, Treadon RE (1997) A tutorial on lateral boundary conditions as a basic and potentially serious limitation to regional numerical weather prediction. Bull Am Meteorol Soc 78: 2599-2617

Yakimiw E, Robert A (1990) Validation experiments for a nested grid-point regional forecast model. Atmos-Ocean 28: 466-472 